\title{
Approximate solutions of a sum-type fractional integro-differential equation by using Chebyshev and Legendre polynomials
}

\author{
Eisa Akbari Kojabad and Shahram Rezapour ${ }^{*}$
}

\section{"Correspondence:}

sh.rezapour@azaruniv.edu Department of Mathematics,

Azarbaijan Shahid Madani

University, Tabriz, Iran

\begin{abstract}
We investigate the existence of solutions for a sum-type fractional integro-differential problem via the Caputo differentiation. By using the shifted Legendre and Chebyshev polynomials, we provide a numerical method for finding solutions for the problem. In this way, we give some examples to illustrate our results.
\end{abstract}

MSC: 26A33; 34A08; 34K37

Keywords: approximate fixed point; Chebyshev polynomial; Legendre polynomial; numerical solution; sum-type fractional integro-differential equation

\section{Introduction}

In 1969, Reinermann investigated some problems by using approximate fixed point property ([1]). In 1976, Yamamoto and Ohtsubo published a paper on subspace iteration accelerated by using Chebyshev polynomials for eigenvalue problems ([2]). There has been published some work about different fractional integro-differential equations by using Chebyshev polynomials ([3, 4] and [5]) or by using Legendre wavelets ([6-8] and [9]). Recently, different techniques for solving some fractional integro-differential equations have been used (see [6, 10-19]). In this paper by using an approximate fixed point result and the shifted Legendre and Chebyshev polynomials, we investigate the existence of solutions for a sum-type fractional integro-differential problem.

As is well known, the Caputo fractional derivative of order $\beta$ for a continuous function $f:(0, \infty) \rightarrow \mathbb{R}$ is defined by ${ }^{c} D^{\beta} f(t)=\frac{1}{\Gamma(n-\beta)} \int_{0}^{t} \frac{f^{(n)}(s)}{(t-s)^{\beta-n+1}} d s$, where $n=[\beta]+1([20,21])$. The fractional integral of order $\beta$ for a function $f:(0, \infty) \rightarrow \mathbb{R}$ is defined by $I^{\beta} f(t)=\frac{1}{\Gamma(\beta)} \int_{0}^{t}(t-$ $s)^{\beta-1} f(s) d s([20,21])$. Let $(X, d)$ be a metric space, $T$ a selfmap on $X$ and $\alpha: X \times X \rightarrow[0, \infty)$ a map. We say that $T$ is $\alpha$-admissible whenever $\alpha(x, y) \geq 1$ implies $\alpha(T x, T y) \geq 1$. Also, $T$ is called $\alpha$-contraction whenever there exists $\lambda \in(0,1)$ such that $\alpha(x, y) d(T x, T y) \leq \lambda d(x, y)$ for all $x, y \in X$. We say that $T$ has approximate fixed point property whenever there exists a sequence $\left\{x_{n}\right\}_{n \geq 1}$ in $X$ such that $d\left(x_{n}, T x_{n}\right) \rightarrow 0$. We need the following results.

Lemma 1.1 ([21]) Let $q>0, n=[q]+1$ and $v \in C([0,1], \mathbb{R})$. Then the fractional differential equation ${ }^{c} D^{q} x(t)=v(t)$ has a solution in the form

$$
x(t)=I^{q} v(t)+c_{0}+c_{1} t+\cdots+c_{n-1} t^{n-1} .
$$

(c) The Author(s) 2017. This article is distributed under the terms of the Creative Commons Attribution 4.0 International License (http://creativecommons.org/licenses/by/4.0/), which permits unrestricted use, distribution, and reproduction in any medium, provided you give appropriate credit to the original author(s) and the source, provide a link to the Creative Commons license, and indicate if changes were made. 
Lemma 1.2 ([22]) Let $(X, d)$ be a metric space and $T$ an $\alpha$-contractive and $\alpha$-admissible selfmap on $X$ such that $\alpha\left(x_{0}, T x_{0}\right) \geq 1$ for some $x_{0} \in X$. Then $T$ has the approximate fixed point property. If $X$ is complete and $T$ is continuous, then $T$ has fixed point.

\section{Main result}

Now, we are ready to study the existence of solution of the sum-type fractional integrodifferential equation

$$
{ }^{c} D^{q} x(t)=f\left(t, x(t),{ }^{c} D^{\beta_{1}} x(t), \ldots,{ }^{c} D^{\beta_{n}} x(t)\right)+g\left(t, x(t), I^{\beta_{1}} x(t), \ldots I^{\beta_{n}} x(t)\right)
$$

with boundary value conditions $\sum_{i=1}^{n}\left(a_{i}{ }^{c} D^{\beta_{i}} x(1)\right)=\alpha_{1} x^{\prime}(1)$ and $\sum_{i=1}^{n}\left(b_{i} I^{\beta_{i}} x(1)\right)=\alpha_{2} x^{\prime}(0)$, where $1<q<2, a_{1}, \ldots, a_{n}, b_{1}, \ldots, b_{n} \in \mathbb{R}$ and $f, g:[0,1] \times \mathbb{R}^{n+2} \rightarrow \mathbb{R}$ are two maps.

Lemma 2.1 Let $1<q<2$ and $v \in C(I, \mathbb{R})$. Then the unique solution for the fractional differential equation ${ }^{c} D^{q} x(t)=v(t)$ with boundary conditions $\sum_{i=1}^{n}\left(a_{i}{ }^{c} D^{\beta_{i}} x(1)\right)=\alpha_{1} x^{\prime}(1)$ and $\sum_{i=1}^{n}\left(b_{i} I^{\beta_{i}} x(1)\right)=\alpha_{2} x^{\prime}(0)$ is given by

$$
\begin{aligned}
x(t)= & I^{q} v(t)-\frac{\left(\sum_{i=1}^{n} \frac{a_{i}}{\Gamma\left(2-\beta_{i}\right)}-\alpha_{1}\right)}{\left(\sum_{i=1}^{n} \frac{a_{i}}{\Gamma\left(2-\beta_{i}\right)}-\alpha_{1}\right)\left(\sum_{i=1}^{n}\left(\frac{b_{i}}{\Gamma\left(\beta_{i}+1\right)}\right)\right)} \sum_{i=1}^{n} b_{i} I^{q+\beta_{i}} v(1) \\
& -\frac{\sum_{i=1}^{n}\left(\frac{b_{i}}{\Gamma\left(\beta_{i}+2\right)}-\alpha_{2}\right)}{\left(\sum_{i=1}^{n} \frac{a_{i}}{\Gamma\left(2-\beta_{i}\right)}-\alpha_{1}\right)\left(\sum_{i=1}^{n}\left(\frac{b_{i}}{\Gamma\left(\beta_{i}+1\right)}\right)\right)} \alpha_{1} I^{q-1} v(1) \\
& +\frac{\sum_{i=1}^{n}\left(\frac{b_{i}}{\Gamma\left(\beta_{i}+2\right)}-\alpha_{2}\right)}{\left(\sum_{i=1}^{n} \frac{a_{i}}{\Gamma\left(2-\beta_{i}\right)}-\alpha_{1}\right)\left(\sum_{i=1}^{n}\left(\frac{b_{i}}{\Gamma\left(\beta_{i}+1\right)}\right)\right)} \sum_{i=1}^{n} a_{i} I^{q-\beta_{i}} v(1) \\
& +\frac{\alpha_{1} t I^{q-1} v(1)-\sum_{i=1}^{n}\left(t a_{i} I^{q-\beta_{i}} v(1)\right)}{\sum_{i=1}^{n} \frac{a_{i}}{\Gamma\left(2-\beta_{i}\right)}-\alpha_{1}}
\end{aligned}
$$

where $\alpha_{1}, \alpha_{2}, a_{1}, \ldots, a_{n}, b_{1}, \ldots, b_{n}$ are some real numbers.

Proof By using Lemma 1.1, general solution for the equation ${ }^{c} D^{q} x(t)=v(t)$ is given by $x(t)=\frac{1}{\Gamma(q)} \int_{0}^{t}(t-s)^{q-1} v(s) d s+c_{0}+c_{1} t$, where $c_{0}, c_{1} \in \mathbb{R}$. By applying the boundary condition $\sum_{i=1}^{n}\left(a_{i}^{c} D^{\beta_{i}} x(1)\right)=\alpha_{1} x^{\prime}(1)$, we get

$$
\begin{aligned}
& \sum_{i=0}^{n}\left(\frac{a_{i}}{\Gamma\left(q-\beta_{i}\right)} \int_{0}^{1}(1-s)^{q-\beta_{i}-1} v(s) d s+\frac{a_{i} c_{1}}{\Gamma\left(2-\beta_{i}\right)}+0\right) \\
& \quad=\frac{\alpha_{1}}{\Gamma(q-1)} \int_{0}^{1}(1-s)^{q-2} v(s) d s+\alpha_{1} c_{1}
\end{aligned}
$$

and by using the boundary condition $\sum_{i=1}^{n}\left(b_{i} I^{\beta_{i}} x(1)\right)=\alpha_{2} x^{\prime}(0)$, we get

$$
\sum_{i=1}^{n}\left(\frac{b_{i}}{\Gamma\left(q+\beta_{i}\right)} \int_{0}^{1}(1-s)^{q+\beta_{i}-1} v(s) d s+\frac{b_{i} c_{0}}{\Gamma\left(\beta_{i}+1\right)}+\frac{b_{i} c_{1}}{\Gamma\left(\beta_{i}+2\right)}\right)=\alpha_{2} c_{1} .
$$


This implies

$$
\begin{aligned}
& c_{1}\left(\sum_{i=1}^{n} \frac{a_{i}}{\Gamma\left(2-\beta_{i}\right)}-\alpha_{1}\right) \\
& \quad=\frac{\alpha_{1}}{\Gamma(q-1)} \int_{0}^{1}(1-s)^{q-2} v(s) d s-\sum_{i=1}^{n}\left(\frac{a_{i}}{\Gamma\left(q-\beta_{i}\right)} \int_{0}^{1}(1-s)^{q-\beta_{i}-1} v(s) d s\right)
\end{aligned}
$$

and

$$
\sum_{i=1}^{n}\left[c_{1}\left(\frac{b_{i}}{\Gamma\left(\beta_{i}+2\right)}-\alpha_{2}\right)+\left(\frac{b_{i}}{\Gamma\left(\beta_{i}+1\right)}\right) c_{0}\right]=-\sum_{i=1}^{n} \frac{b_{i}}{\Gamma\left(q+\beta_{i}\right)} \int_{0}^{1}(1-s)^{q+\beta_{i}-1} v(s) d s
$$

Hence,

$$
\begin{aligned}
c_{0}= & -\frac{\left(\sum_{i=1}^{n} \frac{b_{i}}{\Gamma\left(q+\beta_{i}\right)} \int_{0}^{1}(1-s)^{q+\beta_{i}-1} v(s) d s\right)\left(\sum_{i=1}^{n} \frac{a_{i}}{\Gamma\left(2-\beta_{i}\right)}-\alpha_{1}\right)}{\left(\sum_{i=1}^{n} \frac{a_{i}}{\Gamma\left(2-\beta_{i}\right)}-\alpha_{1}\right)\left(\sum_{i=1}^{n}\left(\frac{b_{i}}{\Gamma\left(\beta_{i}+1\right)}\right)\right)} \\
& -\frac{\left(\sum_{i=1}^{n}\left(\frac{b_{i}}{\Gamma\left(\beta_{i}+2\right)}-\alpha_{2}\right)\right)\left(\frac{\alpha_{1}}{\Gamma(q-1)} \int_{0}^{1}(1-s)^{q-2} v(s) d s\right)}{\left(\sum_{i=1}^{n} \frac{a_{i}}{\Gamma\left(2-\beta_{i}\right)}-\alpha_{1}\right)\left(\sum_{i=1}^{n}\left(\frac{b_{i}}{\Gamma\left(\beta_{i}+1\right)}\right)\right)} \\
& +\frac{\left(\sum_{i=1}^{n}\left(\frac{b_{i}}{\Gamma\left(\beta_{i}+2\right)}-\alpha_{2}\right)\right)\left(\sum_{i=1}^{n}\left(\frac{a_{i}}{\Gamma\left(q-\beta_{i}\right)} \int_{0}^{1}(1-s)^{q-\beta_{i}-1} v(s) d s\right)\right)}{\left(\sum_{i=1}^{n} \frac{a_{i}}{\Gamma\left(2-\beta_{i}\right)}-\alpha_{1}\right)\left(\sum_{i=1}^{n}\left(\frac{b_{i}}{\Gamma\left(\beta_{i}+1\right)}\right)\right)}
\end{aligned}
$$

and

$$
c_{1}=\frac{\frac{\alpha_{1}}{\Gamma(q-1)} \int_{0}^{1}(1-s)^{q-2} v(s) d s-\sum_{i=1}^{n}\left(\frac{a_{i}}{\Gamma\left(q-\beta_{i}\right)} \int_{0}^{1}(1-s)^{q-\beta_{i}-1} v(s) d s\right)}{\sum_{i=1}^{n} \frac{a_{i}}{\Gamma\left(2-\beta_{i}\right)}-\alpha_{1}} .
$$

Thus,

$$
\begin{aligned}
x(t)= & \frac{1}{\Gamma(q)} \int_{0}^{t}(t-s)^{q-1} v(s) d s \\
& -\frac{\left(\sum_{i=1}^{n} \frac{b_{i}}{\Gamma\left(q+\beta_{i}\right)} \int_{0}^{1}(1-s)^{q+\beta_{i}-1} v(s) d s\right)\left(\sum_{i=1}^{n} \frac{a_{i}}{\Gamma\left(2-\beta_{i}\right)}-\alpha_{1}\right)}{\left(\sum_{i=1}^{n} \frac{a_{i}}{\Gamma\left(2-\beta_{i}\right)}-\alpha_{1}\right)\left(\sum_{i=1}^{n}\left(\frac{b_{i}}{\Gamma\left(\beta_{i}+1\right)}\right)\right)} \\
& -\frac{\left(\sum_{i=1}^{n}\left(\frac{b_{i}}{\Gamma\left(\beta_{i}+2\right)}-\alpha_{2}\right)\right)\left(\frac{\alpha_{1}}{\Gamma(q-1)} \int_{0}^{1}(1-s)^{q-2} v(s) d s\right)}{\left(\sum_{i=1}^{n} \frac{a_{i}}{\Gamma\left(2-\beta_{i}\right)}-\alpha_{1}\right)\left(\sum_{i=1}^{n}\left(\frac{b_{i}}{\Gamma\left(\beta_{i}+1\right)}\right)\right)} \\
& +\frac{\left(\sum_{i=1}^{n}\left(\frac{b_{i}}{\Gamma\left(\beta_{i}+2\right)}-\alpha_{2}\right)\right)\left(\sum_{i=1}^{n}\left(\frac{a_{i}}{\Gamma\left(q-\beta_{i}\right)} \int_{0}^{1}(1-s)^{q-\beta_{i}-1} v(s) d s\right)\right)}{\left(\sum_{i=1}^{n} \frac{a_{i}}{\Gamma\left(2-\beta_{i}\right)}-\alpha_{1}\right)\left(\sum_{i=1}^{n}\left(\frac{b_{i}}{\Gamma\left(\beta_{i}+1\right)}\right)\right)} \\
& +\frac{\frac{t \alpha_{1}}{\Gamma(q-1)} \int_{0}^{1}(1-s)^{q-2} v(s) d s-t \sum_{i=1}^{n}\left(\frac{a_{i}}{\Gamma\left(q-\beta_{i}\right)} \int_{0}^{1}(1-s)^{q-\beta_{i}-1} v(s) d s\right)}{\sum_{i=1}^{n} \frac{a_{i}}{\Gamma\left(2-\beta_{i}\right)}-\alpha_{1}} \\
= & I^{q} v(t)-\frac{a_{i}}{\left(\sum_{i=1}^{n} \frac{a_{i}}{\Gamma\left(2-\beta_{i}\right)}-\alpha_{1}\right)\left(\sum_{i=1}^{n}\left(\frac{b_{i}}{\Gamma\left(\beta_{i}+1\right)}\right)\right)} \sum_{i=1}^{n} b_{i} I^{q+\beta_{i}} v(1) \\
& -\frac{\sum_{i=1}^{n}\left(\frac{b_{i}}{\Gamma\left(\beta_{i}+2\right)}-\alpha_{2}\right)}{\left(\sum_{i=1}^{n} \frac{a_{i}}{\Gamma\left(2-\beta_{i}\right)}-\alpha_{1}\right)\left(\sum_{i=1}^{n}\left(\frac{b_{i}}{\Gamma\left(\beta_{i}+1\right)}\right)\right)} \alpha_{1} I^{q-1} v(1)
\end{aligned}
$$




$$
\begin{aligned}
& +\frac{\sum_{i=1}^{n}\left(\frac{b_{i}}{\Gamma\left(\beta_{i}+2\right)}-\alpha_{2}\right)}{\left(\sum_{i=1}^{n} \frac{a_{i}}{\Gamma\left(2-\beta_{i}\right)}-\alpha_{1}\right)\left(\sum_{i=1}^{n}\left(\frac{b_{i}}{\Gamma\left(\beta_{i}+1\right)}\right)\right)} \sum_{i=1}^{n} a_{i} I^{q-\beta_{i}} v(1) \\
& +\frac{\alpha_{1} t I^{q-1} v(1)-\sum_{i=1}^{n}\left(t a_{i} I^{q-\beta_{i}} v(1)\right)}{\sum_{i=1}^{n} \frac{a_{i}}{\Gamma\left(2-\beta_{i}\right)}-\alpha_{1}} .
\end{aligned}
$$

One can check that the given $x(t)$ is a solution for the problem ${ }^{c} D^{q} x(t)=v(t)$ with the boundary conditions. This completes our proof.

Let $\mathcal{X}=\left\{x: x,{ }^{c} D^{\beta_{1}} x,{ }^{c} D^{\beta_{2}} x, \ldots,{ }^{c} D^{\beta_{n}} x \in C(I, \mathbb{R})\right\}$ be endowed with the metric

$$
d(x, y)=\sup _{t \in I}|x(t)-y(t)|+\sup _{t \in I}\left|{ }^{c} D^{\beta_{1}} x(t)-{ }^{c} D^{\beta_{1}} y(t)\right|+\cdots+\sup _{t \in I}\left|{ }^{c} D^{\beta_{n}} x(t)-{ }^{c} D^{\beta_{n}} y(t)\right| .
$$

It is clear that $(\mathcal{X}, d)$ is a complete metric space (see [23]). By using Lemma 2.1, a function $x \in \mathcal{X}$ is a solution for the fractional differential equation (2.1) whenever it satisfies the boundary conditions and there exist functions $v, v^{\prime} \in L^{1}[0,1]$ such that $v(t)=$ $f\left(t, x(t),{ }^{c} D^{\beta_{1}} x(t), \ldots,{ }^{c} D^{\beta_{n}} x(t)\right), v^{\prime}(t)=g\left(t, x(t), I^{\beta_{1}} x(t), \ldots, I^{\beta_{n}} x(t)\right)$ and

$$
\begin{aligned}
x(t)= & I^{q}\left(v(t)+v^{\prime}(t)\right) \\
& -\frac{\left(\sum_{i=1}^{n} \frac{a_{i}}{\Gamma\left(2-\beta_{i}\right)}-\alpha_{1}\right)}{\left(\sum_{i=1}^{n} \frac{a_{i}}{\Gamma\left(2-\beta_{i}\right)}-\alpha_{1}\right)\left(\sum_{i=1}^{n}\left(\frac{b_{i}}{\Gamma\left(\beta_{i}+1\right)}\right)\right)} \sum_{i=1}^{n} b_{i} I^{q+\beta_{i}}\left(v(1)+v^{\prime}(1)\right) \\
& -\frac{\sum_{i=1}^{n}\left(\frac{b_{i}}{\Gamma\left(\beta_{i}+2\right)}-\alpha_{2}\right)}{\left(\sum_{i=1}^{n} \frac{a_{i}}{\Gamma\left(2-\beta_{i}\right)}-\alpha_{1}\right)\left(\sum_{i=1}^{n}\left(\frac{b_{i}}{\Gamma\left(\beta_{i}+1\right)}\right)\right)} \alpha_{1} I^{q-1}\left(v(1)+v^{\prime}(1)\right) \\
& +\frac{\sum_{i=1}^{n}\left(\frac{b_{i}}{\Gamma\left(\beta_{i}+2\right)}-\alpha_{2}\right)}{\left(\sum_{i=1}^{n} \frac{a_{i}}{\Gamma\left(2-\beta_{i}\right)}-\alpha_{1}\right)\left(\sum_{i=1}^{n}\left(\frac{b_{i}}{\Gamma\left(\beta_{i}+1\right)}\right)\right)} \sum_{i=1}^{n} a_{i} I^{q-\beta_{i}}\left(v(1)+v^{\prime}(1)\right) \\
& +\frac{\alpha_{1} t I^{q-1}\left(\nu(1)+v^{\prime}(1)\right)-\sum_{i=1}^{n}\left(t a_{i} I^{q-\beta_{i}}\left(v(1)+v^{\prime}(1)\right)\right)}{\sum_{i=1}^{n} \frac{a_{i}}{\Gamma\left(2-\beta_{i}\right)}-\alpha_{1}}
\end{aligned}
$$

for all $t \in I$.

Theorem 2.2 Let $\xi: \mathbb{R}^{2(n+1)} \rightarrow \mathbb{R}$ be a map, $\lambda \in(0,1)$ and $f, g:[0,1] \times \mathbb{R}^{n+2} \rightarrow \mathbb{R}$ two functions such that

$$
\begin{aligned}
& \left|f\left(t, x_{1}, x_{2}, \ldots, x_{n+1}\right)-f\left(t, y_{1}, y_{2}, \ldots, y_{n+1}\right)\right|+\left|g\left(t, x_{1}, x_{2}, \ldots, x_{n+1}\right)-g\left(t, y_{1}, y_{2}, \ldots, y_{n+1}\right)\right| \\
& \quad \leq \frac{\lambda}{\Omega_{1}+n \Omega_{2}}\left(\left|x_{1}-y_{1}\right|+\cdots+\left|x_{n+1}-y_{n+1}\right|\right)
\end{aligned}
$$

for all $t \in I=[0,1]$ and $x_{1}, \ldots, x_{n}, y_{1}, \ldots, y_{n} \in \mathbb{R}$ with

$$
\xi\left(x_{1}, x_{2}, \ldots, x_{n+1}, y_{1}, y_{2}, \ldots, y_{n+1}\right) \geq 0,
$$

where

$$
\begin{aligned}
\Omega_{1}= & {\left[\left|\frac{1}{\Gamma(q+1)}\right|+\frac{\left|\frac{\alpha_{1}}{\Gamma(q)}\right|+\left.\sum_{i=1}^{n}\right|_{i} ^{5} \lambda \mid}{\left|\sum_{i=1}^{n} \lambda_{i}^{1}-\alpha_{1}\right|}\right.} \\
& \left.+\frac{\left|\sum_{i=1}^{n} \lambda_{i}^{1}-\alpha_{1}\right|\left|\sum_{i=1}^{n}{ }_{i}^{3} \lambda\right|+\left|\sum_{i=1}^{n} \lambda_{i}^{4}-\alpha_{2}\right|\left(\left|\frac{\alpha_{1}}{\Gamma(q)}\right|+\left|\sum_{i=1}^{n}{ }_{i}^{5} \lambda\right|\right)}{\left|\left(\sum_{i=1}^{n} \lambda_{i}^{1}-\alpha_{1}\right) \sum_{i=1}^{n} \lambda_{i}^{2}\right|}\right],
\end{aligned}
$$




$$
\begin{aligned}
& \Omega_{2}=\max _{1 \leq j \leq n}\left(\left|\frac{1}{\Gamma\left(q-\beta_{j}+1\right)}\right|+\left|\frac{\frac{\alpha_{1}}{\Gamma(q)}}{\Gamma\left(2-\beta_{j}\right)\left(\sum_{i=1}^{n} \lambda_{i}^{1}-\alpha_{1}\right)}\right|\right), \\
& \lambda_{i}^{1}=\frac{a_{i}}{1-\Gamma\left(2-\beta_{i}\right)}, \quad \lambda_{i}^{2}=\frac{b_{i}}{\Gamma\left(\beta_{i}+1\right)}, \quad \lambda_{i}^{3}=\frac{b_{i}}{\Gamma\left(q+\beta_{i}\right)}, \quad{ }_{i}^{3} \lambda=\frac{b_{i}}{\Gamma\left(q+\beta_{i}+1\right)}, \\
& \lambda_{i}^{4}=\frac{b_{i}}{\Gamma\left(\beta_{i}+2\right)}, \quad \lambda_{i}^{5}=\frac{a_{i}}{\Gamma\left(q-\beta_{i}\right)} \quad \text { and } \quad{ }_{i}^{5} \lambda=\frac{a_{i}}{\Gamma\left(q-\beta_{i}+1\right)} .
\end{aligned}
$$

Assume that

$$
\xi\left(u(t),{ }^{c} D^{\beta_{1}} u(t),{ }^{c} D^{\beta_{2}} u(t), \ldots,{ }^{c} D^{\beta_{n}} u(t), v(t),{ }^{c} D^{\beta_{1}} v(t),{ }^{c} D^{\beta_{2}} v(t), \ldots,{ }^{c} D^{\beta_{n}} v(t)\right) \geq 0
$$

implies

$$
\xi\left(T u(t),{ }^{c} D^{\beta_{1}} T u(t), \ldots,{ }^{c} D^{\beta_{n}} T u(t), T v(t),{ }^{c} D^{\beta_{1}} T v(t), \ldots,{ }^{c} D^{\beta_{n}} T v(t)\right) \geq 0,
$$

where the operator $T: \mathcal{X} \rightarrow \mathcal{X}$ is defined by

$$
\begin{aligned}
T u(t)= & \frac{1}{\Gamma(q)} \int_{0}^{t}(t-s)^{q-1} f\left(s, u(s),{ }^{c} D^{\beta_{1}} u(s), \ldots,{ }^{c} D^{\beta_{n}} u(s)\right) d s \\
& -\left[\frac{\sum_{i=1}^{n} \lambda_{i}^{1}-\alpha_{1}}{\left(\sum_{i=1}^{n} \lambda_{i}^{1}-\alpha_{1}\right) \sum_{i=1}^{n} \lambda_{i}^{2}}\right] \\
& \times \sum_{i=1}^{n}\left(\lambda_{i}^{3} \int_{0}^{1}(1-s)^{q+\beta_{i}-1} f\left(s, u(s),{ }^{c} D^{\beta_{1}} u(s), \ldots,{ }^{c} D^{\beta_{n}} u(s)\right) d s\right) \\
& -\left[\frac{\sum_{i=1}^{n} \lambda_{i}^{4}-\alpha_{2}}{\left(\sum_{i=1}^{n} \lambda_{i}^{1}-\alpha_{1}\right)\left(\sum_{i=1}^{n} \lambda_{i}^{2}\right)}\right] \\
& \times \frac{\alpha_{1}}{\Gamma(q-1)} \int_{0}^{1}(1-s)^{q-2} f\left(s, u(s),{ }^{c} D^{\beta_{1}} u(s), \ldots,{ }^{c} D^{\beta_{n}} u(s)\right) d s \\
& -\left[\frac{\sum_{i=1}^{n} \lambda_{i}^{4}-\alpha_{2}}{\left(\sum_{i=1}^{n} \lambda_{i}^{1}-\alpha_{1}\right) \sum_{i=1}^{n} \lambda_{i}^{2}}\right] \\
& \times \sum_{i=1}^{n}\left(\lambda_{i}^{5} \int_{0}^{1}(1-s)^{q-\beta_{i}-1} f\left(s, u(s),{ }^{c} D^{\beta_{1}} u(s), \ldots,{ }^{c} D^{\beta_{n}} u(s)\right) d s\right) \\
& +\frac{t \alpha_{1}}{\Gamma(q-1)} \int_{0}^{1}(1-s)^{q-2} f\left(s, u(s),{ }^{c} D^{\beta_{1}} u(s), \ldots,{ }^{c} D^{\beta_{n}} u(s)\right) d s \\
& -\left[\frac{\sum_{i=1}^{n} \lambda_{i}^{4}-\alpha_{2}}{\left(\sum_{i=1}^{n} \lambda_{i}^{1}-\alpha_{1}\right)\left(\sum_{i=1}^{n} \lambda_{i}^{2}\right)}\right] \\
& -\frac{t \sum_{i=1}^{n}\left(\lambda_{i}^{5} \int_{0}^{1}(1-s)^{q-\beta_{i}-1} f\left(s, u(s),{ }^{c} D^{\beta_{1}} u(s), \ldots,{ }^{c} D^{\beta_{n}} u(s)\right) d s\right)}{\sum_{i=1}^{n} \lambda_{i}^{1}-\alpha_{1}} \\
& +\frac{1}{\Gamma(q)} \int_{0}^{t}(t-s)^{q-1} g\left(s, u(s), I^{\beta_{1}} u(s), \ldots, I^{\beta_{n}} u(s)\right) d s \\
& \left.-\left[\sum_{i=1}^{n} \lambda_{i}^{n}-\alpha_{1} \lambda_{i}^{1}-\alpha_{1}\right) \sum_{i=1}^{n} \int_{i}^{1}(1-s)^{q+\beta_{i}-1} g\left(s, u(s), I^{\beta_{1}} u(s), \ldots, I^{\beta_{n}} u(s)\right) d s\right) \\
& \\
&
\end{aligned}
$$




$$
\begin{aligned}
& \times \frac{\alpha_{1}}{\Gamma(q-1)} \int_{0}^{1}(1-s)^{q-2} g\left(s, u(s), I^{\beta_{1}} u(s), \ldots, I^{\beta_{n}} u(s)\right) d s \\
& -\left[\frac{\sum_{i=1}^{n} \lambda_{i}^{4}-\alpha_{2}}{\left(\sum_{i=1}^{n} \lambda_{i}^{1}-\alpha_{1}\right) \sum_{i=1}^{n} \lambda_{i}^{2}}\right] \\
& \times \sum_{i=1}^{n}\left(\lambda_{i}^{5} \int_{0}^{1}(1-s)^{q-\beta_{i}-1} g\left(s, u(s), I^{\beta_{1}} u(s), \ldots, I^{\beta_{n}} u(s)\right) d s\right) \\
& +\frac{\frac{t \alpha_{1}}{\Gamma(q-1)} \int_{0}^{1}(1-s)^{q-2} g\left(s, u(s), I^{\beta_{1}} u(s), \ldots, I^{\beta_{n}} u(s)\right) d s}{\sum_{i=1}^{n} \lambda_{i}^{1}-\alpha_{1}} \\
& -\frac{t \sum_{i=1}^{n}\left(\lambda_{i}^{5} \int_{0}^{1}(1-s)^{q-\beta_{i}-1} g\left(s, u(s), I^{\beta_{1}} u(s), \ldots, I^{\beta_{n}} u(s)\right) d s\right)}{\sum_{i=1}^{n} \lambda_{i}^{1}-\alpha_{1}}
\end{aligned}
$$

for all $t \in I$. If there exists $u_{1} \in \mathcal{X}$ such that

$$
\xi\left(u_{1}(t),{ }^{c} D^{\beta_{1}} u_{1}(t), \ldots,{ }^{c} D^{\beta_{n}} u_{1}(t), T u_{1}(t),{ }^{c} D^{\beta_{1}} T u_{1}(t), \ldots,{ }^{c} D^{\beta_{n}} T u_{1}(t)\right) \geq 0
$$

for all $t \in[0,1]$, then the problem (2.1) has an approximate solution.

Proof We define $\alpha: \mathcal{X} \times \mathcal{X} \rightarrow[0, \infty)$ by

$$
\alpha(u, v)= \begin{cases}1, & \left.\xi\left(u(t),{ }^{c} D^{\beta_{1}} u(t), \ldots,{ }^{c} D^{\beta_{n}} u(t), v(t),{ }^{c} D^{\beta_{1}} v(t)\right), \ldots,{ }^{c} D^{\beta_{n}} v(t)\right) \geq 0, \forall t \in I, \\ 0, & \text { else. }\end{cases}
$$

We show that $T$ is an $\alpha$-admissible and $\alpha$-contractive selfmap on $\mathcal{X}$. Let $u, v \in \mathcal{X}$ be such that $\xi\left(u(t),{ }^{c} D^{\beta_{1}} u(t), \ldots,{ }^{c} D^{\beta_{n}} u(t), v(t),{ }^{c} D^{\beta_{1}} v(t), \ldots,{ }^{c} D^{\beta_{n}} v(t)\right) \geq 0$ for all $t \in[0,1]$. Then we have

$$
\begin{aligned}
\mid T u(t) & -T v(t) \mid \\
=\mid & \left\{\frac{1}{\Gamma(q)} \int_{0}^{t}(t-s)^{q-1} f\left(s, u(s),{ }^{c} D^{\beta_{1}} u(s), \ldots,{ }^{c} D^{\beta_{n}} u(s)\right) d s\right. \\
& -\left[\frac{\sum_{i=1}^{n} \lambda_{i}^{1}-\alpha_{1}}{\left(\sum_{i=1}^{n} \lambda_{i}^{1}-\alpha_{1}\right) \sum_{i=1}^{n} \lambda_{i}^{2}}\right] \\
& \times \sum_{i=1}^{n}\left(\lambda_{i}^{3} \int_{0}^{1}(1-s)^{q+\beta_{i}-1} f\left(s, u(s),{ }^{c} D^{\beta_{1}} u(s), \ldots,{ }^{c} D^{\beta_{n}} u(s)\right) d s\right) \\
& -\left[\frac{\sum_{i=1}^{n} \lambda_{i}^{4}-\alpha_{2}}{\left(\sum_{i=1}^{n} \lambda_{i}^{1}-\alpha_{1}\right)\left(\sum_{i=1}^{n} \lambda_{i}^{2}\right)}\right] \\
& \times \frac{\alpha_{1}}{\Gamma(q-1)} \int_{0}^{1}(1-s)^{q-2} f\left(s, u(s),{ }^{c} D^{\beta_{1}} u(s), \ldots,{ }^{c} D^{\beta_{n}} u(s)\right) d s \\
& -\left[\frac{\sum_{i=1}^{n} \lambda_{i}^{4}-\alpha_{2}}{\left(\sum_{i=1}^{n} \lambda_{i}^{1}-\alpha_{1}\right) \sum_{i=1}^{n} \lambda_{i}^{2}}\right] \\
& \times \sum_{i=1}^{n}\left(\lambda_{i}^{5} \int_{0}^{1}(1-s)^{q-\beta_{i}-1} f\left(s, u(s),{ }^{c} D^{\beta_{1}} u(s), \ldots,{ }^{c} D^{\beta_{n}} u(s)\right) d s\right)
\end{aligned}
$$




$$
\begin{aligned}
& +\frac{\frac{t \alpha_{1}}{\Gamma(q-1)} \int_{0}^{1}(1-s)^{q-2} f\left(s, u(s),{ }^{c} D^{\beta_{1}} u(s), \ldots,{ }^{c} D^{\beta_{n}} u(s)\right) d s}{\sum_{i=1}^{n} \lambda_{i}^{1}-\alpha_{1}} \\
& -\frac{t \sum_{i=1}^{n}\left(\lambda_{i}^{5} \int_{0}^{1}(1-s)^{q-\beta_{i}-1} f\left(s, u(s),{ }^{c} D^{\beta_{1}} u(s), \ldots,{ }^{c} D^{\beta_{n}} u(s)\right) d s\right)}{\sum_{i=1}^{n} \lambda_{i}^{1}-\alpha_{1}} \\
& +\frac{1}{\Gamma(q)} \int_{0}^{t}(t-s)^{q-1} g\left(s, u(s), I^{\beta_{1}} u(s), \ldots, I^{\beta_{n}} u(s)\right) d s \\
& -\left[\frac{\sum_{i=1}^{n} \lambda_{i}^{1}-\alpha_{1}}{\left(\sum_{i=1}^{n} \lambda_{i}^{1}-\alpha_{1}\right) \sum_{i=1}^{n} \lambda_{i}^{2}}\right] \\
& \times \sum_{i=1}^{n}\left(\lambda_{i}^{3} \int_{0}^{1}(1-s)^{q+\beta_{i}-1} g\left(s, u(s), I^{\beta_{1}} u(s), \ldots, I^{\beta_{n}} u(s)\right) d s\right) \\
& -\left[\frac{\sum_{i=1}^{n} \lambda_{i}^{4}-\alpha_{2}}{\left(\sum_{i=1}^{n} \lambda_{i}^{1}-\alpha_{1}\right)\left(\sum_{i=1}^{n} \lambda_{i}^{2}\right)}\right] \frac{\alpha_{1}}{\Gamma(q-1)} \int_{0}^{1}(1-s)^{q-2} g\left(s, u(s), I^{\beta_{1}} u(s), \ldots, I^{\beta_{n}} u(s)\right) d s \\
& -\left[\frac{\sum_{i=1}^{n} \lambda_{i}^{4}-\alpha_{2}}{\left(\sum_{i=1}^{n} \lambda_{i}^{1}-\alpha_{1}\right) \sum_{i=1}^{n} \lambda_{i}^{2}}\right] \\
& \times \sum_{i=1}^{n}\left(\lambda_{i}^{5} \int_{0}^{1}(1-s)^{q-\beta_{i}-1} g\left(s, u(s), I^{\beta_{1}} u(s), \ldots, I^{\beta_{n}} u(s)\right) d s\right) \\
& +\frac{\frac{t \alpha_{1}}{\Gamma(q-1)} \int_{0}^{1}(1-s)^{q-2} g\left(s, u(s), I^{\beta_{1}} u(s), \ldots, I^{\beta_{n}} u(s)\right) d s}{\sum_{i=1}^{n} \lambda_{i}^{1}-\alpha_{1}} \\
& \left.-\frac{t \sum_{i=1}^{n}\left(\lambda_{i}^{5} \int_{0}^{1}(1-s)^{q-\beta_{i}-1} g\left(s, u(s), I^{\beta_{1}} u(s), \ldots, I^{\beta_{n}} u(s)\right) d s\right)}{\sum_{i=1}^{n} \lambda_{i}^{1}-\alpha_{1}}\right\} \\
& -\left\{\frac{1}{\Gamma(q)} \int_{0}^{t}(t-s)^{q-1} f\left(s, v(s),{ }^{c} D^{\beta_{1}} v(s), \ldots,{ }^{c} D^{\beta_{n}} v(s)\right) d s\right. \\
& -\left[\frac{\sum_{i=1}^{n} \lambda_{i}^{1}-\alpha_{1}}{\left(\sum_{i=1}^{n} \lambda_{i}^{1}-\alpha_{1}\right) \sum_{i=1}^{n} \lambda_{i}^{2}}\right] \\
& \times \sum_{i=1}^{n}\left(\lambda_{i}^{3} \int_{0}^{1}(1-s)^{q+\beta_{i}-1} f\left(s, v(s),{ }^{c} D^{\beta_{1}} v(s), \ldots,{ }^{c} D^{\beta_{n}} v(s)\right) d s\right) \\
& -\left[\frac{\sum_{i=1}^{n} \lambda_{i}^{4}-\alpha_{2}}{\left(\sum_{i=1}^{n} \lambda_{i}^{1}-\alpha_{1}\right)\left(\sum_{i=1}^{n} \lambda_{i}^{2}\right)}\right] \\
& \times \frac{\alpha_{1}}{\Gamma(q-1)} \int_{0}^{1}(1-s)^{q-2} f\left(s, v(s),{ }^{c} D^{\beta_{1}} v(s), \ldots,{ }^{c} D^{\beta_{n}} v(s)\right) d s \\
& -\left[\frac{\sum_{i=1}^{n} \lambda_{i}^{4}-\alpha_{2}}{\left(\sum_{i=1}^{n} \lambda_{i}^{1}-\alpha_{1}\right) \sum_{i=1}^{n} \lambda_{i}^{2}}\right] \\
& \times \sum_{i=1}^{n}\left(\lambda_{i}^{5} \int_{0}^{1}(1-s)^{q-\beta_{i}-1} f\left(s, v(s),{ }^{c} D^{\beta_{1}} v(s), \ldots,{ }^{c} D^{\beta_{n}} v(s)\right) d s\right) \\
& +\frac{\frac{t \alpha_{1}}{\Gamma(q-1)} \int_{0}^{1}(1-s)^{q-2} f\left(s, v(s),{ }^{c} D^{\beta_{1}} v(s), \ldots,{ }^{c} D^{\beta_{n}} v(s)\right) d s}{\sum_{i=1}^{n} \lambda_{i}^{1}-\alpha_{1}} \\
& -\frac{t \sum_{i=1}^{n}\left(\lambda_{i}^{5} \int_{0}^{1}(1-s)^{q-\beta_{i}-1} f\left(s, v(s),{ }^{c} D^{\beta_{1}} v(s), \ldots,{ }^{c} D^{\beta_{n}} v(s)\right) d s\right)}{\sum_{i=1}^{n} \lambda_{i}^{1}-\alpha_{1}}
\end{aligned}
$$




$$
\begin{aligned}
& +\frac{1}{\Gamma(q)} \int_{0}^{t}(t-s)^{q-1} g\left(s, v(s), I^{\beta_{1}} v(s), \ldots, I^{\beta_{n}} v(s)\right) d s \\
& -\left[\frac{\sum_{i=1}^{n} \lambda_{i}^{1}-\alpha_{1}}{\left(\sum_{i=1}^{n} \lambda_{i}^{1}-\alpha_{1}\right) \sum_{i=1}^{n} \lambda_{i}^{2}}\right] \\
& \times \sum_{i=1}^{n}\left(\lambda_{i}^{3} \int_{0}^{1}(1-s)^{q+\beta_{i}-1} g\left(s, v(s), I^{\beta_{1}} v(s), \ldots, I^{\beta_{n}} v(s)\right) d s\right) \\
& -\left[\frac{\sum_{i=1}^{n} \lambda_{i}^{4}-\alpha_{2}}{\left(\sum_{i=1}^{n} \lambda_{i}^{1}-\alpha_{1}\right)\left(\sum_{i=1}^{n} \lambda_{i}^{2}\right)}\right] \frac{\alpha_{1}}{\Gamma(q-1)} \int_{0}^{1}(1-s)^{q-2} g\left(s, v(s), I^{\beta_{1}} v(s), \ldots, I^{\beta_{n}} v(s)\right) d s \\
& -\left[\frac{\sum_{i=1}^{n} \lambda_{i}^{4}-\alpha_{2}}{\left(\sum_{i=1}^{n} \lambda_{i}^{1}-\alpha_{1}\right) \sum_{i=1}^{n} \lambda_{i}^{2}}\right] \\
& \times \sum_{i=1}^{n}\left(\lambda_{i}^{5} \int_{0}^{1}(1-s)^{q-\beta_{i}-1} g\left(s, v(s), I^{\beta_{1}} v(s), \ldots, I^{\beta_{n}} v(s)\right) d s\right) \\
& +\frac{\frac{t \alpha_{1}}{\Gamma(q-1)} \int_{0}^{1}(1-s)^{q-2} g\left(s, v(s), I^{\beta_{1}} v(s), \ldots, I^{\beta_{n}} v(s)\right) d s}{\sum_{i=1}^{n} \lambda_{i}^{1}-\alpha_{1}} \\
& \left.-\frac{t \sum_{i=1}^{n}\left(\lambda_{i}^{5} \int_{0}^{1}(1-s)^{q-\beta_{i}-1} g\left(s, v(s), I^{\beta_{1}} v(s), \ldots, I^{\beta_{n}} v(s)\right) d s\right)}{\sum_{i=1}^{n} \lambda_{i}^{1}-\alpha_{1}}\right\} \mid \\
& \leq \int_{0}^{t}\left|\frac{(t-s)^{q-1}}{\Gamma(q)}\right| \\
& \times\left|f\left(s, u(s),{ }^{c} D^{\beta_{1}} u(s), \ldots,{ }^{c} D^{\beta_{n}} u(s)\right)-f\left(s, v(s),{ }^{c} D^{\beta_{1}} v(s), \ldots,{ }^{c} D^{\beta_{n}} v(s)\right)\right| d s \\
& +\left|\frac{\sum_{i=1}^{n} \lambda_{i}^{1}-\alpha_{1}}{\left(\sum_{i=1}^{n} \lambda_{i}^{1}-\alpha_{1}\right) \sum_{i=1}^{n} \lambda_{i}^{2}}\right| \\
& \times \sum_{i=1}^{n}\left(\left|\lambda_{i}^{3}\right| \int_{0}^{1}(1-s)^{q+\beta_{i}-1} \mid f\left(s, u(s),{ }^{c} D^{\beta_{1}} u(s), \ldots,{ }^{c} D^{\beta_{n}} u(s)\right)\right. \\
& \left.-f\left(s, v(s),{ }^{c} D^{\beta_{1}} v(s), \ldots,{ }^{c} D^{\beta_{n}} v(s)\right) \mid d s\right) \\
& +\left|\frac{\sum_{i=1}^{n} \lambda_{i}^{4}-\alpha_{2}}{\left(\sum_{i=1}^{n} \lambda_{i}^{1}-\alpha_{1}\right)\left(\sum_{i=1}^{n} \lambda_{i}^{2}\right)}\right| \frac{\alpha_{1}}{\Gamma(q-1)} \int_{0}^{1}(1-s)^{q-2} \mid f\left(s, u(s),{ }^{c} D^{\beta_{1}} u(s), \ldots,{ }^{c} D^{\beta_{n}} u(s)\right) \\
& -f\left(s, v(s),{ }^{c} D^{\beta_{1}} v(s), \ldots,{ }^{c} D^{\beta_{n}} v(s)\right) \mid d s \\
& +\left|\frac{\sum_{i=1}^{n} \lambda_{i}^{4}-\alpha_{2}}{\left(\sum_{i=1}^{n} \lambda_{i}^{1}-\alpha_{1}\right) \sum_{i=1}^{n} \lambda_{i}^{2}}\right| \sum_{i=1}^{n}\left(\lambda_{i}^{5} \int_{0}^{1}(1-s)^{q-\beta_{i}-1} \mid f\left(s, u(s),{ }^{c} D^{\beta_{1}} u(s), \ldots,{ }^{c} D^{\beta_{n}} u(s)\right)\right. \\
& \left.-f\left(s, v(s),{ }^{c} D^{\beta_{1}} v(s), \ldots,{ }^{c} D^{\beta_{n}} v(s)\right) \mid d s\right) \\
& +\left|\frac{\frac{t \alpha_{1}}{\Gamma(q-1)}}{\sum_{i=1}^{n} \lambda_{i}^{1}-\alpha_{1}}\right| \int_{0}^{1}(1-s)^{q-\beta_{i}-1} \mid f\left(s, u(s),{ }^{c} D^{\beta_{1}} u(s), \ldots,{ }^{c} D^{\beta_{n}} u(s)\right) \\
& -f\left(s, v(s),{ }^{c} D^{\beta_{1}} v(s), \ldots,{ }^{c} D^{\beta_{n}} v(s)\right) \mid d s \\
& +\left|\frac{t \sum_{i=1}^{n} \lambda_{i}^{5}}{\sum_{i=1}^{n} \lambda_{i}^{1}-\alpha_{1}}\right| \int_{0}^{1}(1-s)^{q-\beta_{i}-1} \mid f\left(s, u(s),{ }^{c} D^{\beta_{1}} u(s), \ldots,{ }^{c} D^{\beta_{n}} u(s)\right) \\
& -f\left(s, v(s),{ }^{c} D^{\beta_{1}} v(s), \ldots,{ }^{c} D^{\beta_{n}} v(s)\right) \mid d s \\
& +\int_{0}^{t}\left|\frac{(t-s)^{q-1}}{\Gamma(q)}\right| g\left(s, u(s), I^{\beta_{1}} u(s), \ldots, I^{\beta_{n}} u(s)\right)-g\left(s, v(s), I^{\beta_{1}} v(s), \ldots, I^{\beta_{n}} v(s)\right) \mid d s
\end{aligned}
$$




$$
\begin{aligned}
& +\left|\frac{\sum_{i=1}^{n} \lambda_{i}^{1}-\alpha_{1}}{\left(\sum_{i=1}^{n} \lambda_{i}^{1}-\alpha_{1}\right) \sum_{i=1}^{n} \lambda_{i}^{2}}\right| \sum_{i=1}^{n}\left(\left|\lambda_{i}^{3}\right| \int_{0}^{1}(1-s)^{q+\beta_{i}-1} \mid g\left(s, u(s), I^{\beta_{1}} u(s), \ldots, I^{\beta_{n}} u(s)\right)\right. \\
& \left.-g\left(s, v(s), I^{\beta_{1}} v(s), \ldots, I^{\beta_{n}} v(s)\right) \mid d s\right) \\
& +\left|\frac{\sum_{i=1}^{n} \lambda_{i}^{4}-\alpha_{2}}{\left(\sum_{i=1}^{n} \lambda_{i}^{1}-\alpha_{1}\right)\left(\sum_{i=1}^{n} \lambda_{i}^{2}\right)}\right| \frac{\alpha_{1}}{\Gamma(q-1)} \int_{0}^{1}(1-s)^{q-2} \mid g\left(s, u(s), I^{\beta_{1}} u(s), \ldots, I^{\beta_{n}} u(s)\right) \\
& -g\left(s, v(s), I^{\beta_{1}} v(s), \ldots, I^{\beta_{n}} v(s)\right) \mid d s \\
& +\left|\frac{\sum_{i=1}^{n} \lambda_{i}^{4}-\alpha_{2}}{\left(\sum_{i=1}^{n} \lambda_{i}^{1}-\alpha_{1}\right) \sum_{i=1}^{n} \lambda_{i}^{2}}\right| \sum_{i=1}^{n}\left(\lambda_{i}^{5} \int_{0}^{1}(1-s)^{q-\beta_{i}-1} \mid g\left(s, u(s), I^{\beta_{1}} u(s), \ldots, I^{\beta_{n}} u(s)\right)\right. \\
& \left.-g\left(s, v(s), I^{\beta_{1}} v(s), \ldots, I^{\beta_{n}} v(s)\right) \mid d s\right) \\
& \left.+\left|\frac{t \alpha_{1}}{\Gamma(q-1)}\right| \int_{i=1}^{1} \lambda_{i}^{1}-\alpha_{1} \mid 1-s\right)^{q-\beta_{i}-1} \mid g\left(s, u(s), I^{\beta_{1}} u(s), \ldots, I^{\beta_{n}} u(s)\right) \\
& -g\left(s, v(s), I^{\beta_{1}} v(s), \ldots, I^{\beta_{n}} v(s)\right) \mid d s \\
& +\left|\frac{t \sum_{i=1}^{n} \lambda_{i}^{5}}{\sum_{i=1}^{n} \lambda_{i}^{1}-\alpha_{1}}\right| \int_{0}^{1}(1-s)^{q-\beta_{i}-1} \mid g\left(s, u(s), I^{\beta_{1}} u(s), \ldots, I^{\beta_{n}} u(s)\right) \\
& -g\left(s, v(s), I^{\beta_{1}} v(s), \ldots, I^{\beta_{n}} v(s)\right) \mid d s \\
& \leq\left[\left|\frac{1}{\Gamma(q+1)}\right|+\frac{\left|\frac{\alpha_{1}}{\Gamma(q)}\right|+\left.\sum_{i=1}^{n}\right|_{i} ^{5} \lambda \mid}{\left|\sum_{i=1}^{n} \lambda_{i}^{1}-\alpha_{1}\right|}\right. \\
& \left.+\frac{\left|\sum_{i=1}^{n} \lambda_{i}^{1}-\alpha_{1}\right|\left|\sum_{i=1}^{n}{ }_{i}^{3} \lambda\right|+\left|\sum_{i=1}^{n} \lambda_{i}^{4}-\alpha_{2}\right|\left(\left|\frac{\alpha_{1}}{\Gamma(q)}\right|+\left|\sum_{i=1}^{n}{ }_{i}^{5} \lambda\right|\right)}{\left|\left(\sum_{i=1}^{n} \lambda_{i}^{1}-\alpha_{1}\right) \sum_{i=1}^{n} \lambda_{i}^{2}\right|}\right] \\
& \times\left(\sup _{t \in I}\left|f\left(t, u(t),{ }^{c} D^{\beta_{1}} u(t), \ldots,{ }^{c} D^{\beta_{n}} u(t)\right)-f\left(t, v(t),{ }^{c} D^{\beta_{1}} v(t), \ldots,{ }^{c} D^{\beta_{n}} v(t)\right)\right|\right) \\
& +\left[\left|\frac{1}{\Gamma(q+1)}\right|+\frac{\left|\frac{\alpha_{1}}{\Gamma(q)}\right|+\left.\sum_{i=1}^{n}\right|_{i} ^{5} \lambda \mid}{\left|\sum_{i=1}^{n} \lambda_{i}^{1}-\alpha_{1}\right|}\right. \\
& \left.+\frac{\left|\sum_{i=1}^{n} \lambda_{i}^{1}-\alpha_{1}\right|\left|\sum_{i=1}^{n}{ }_{i}^{3} \lambda\right|+\left|\sum_{i=1}^{n} \lambda_{i}^{4}-\alpha_{2}\right|\left(\left|\frac{\alpha_{1}}{\Gamma(q)}\right|+\left|\sum_{i=1}^{n}{ }_{i}^{5} \lambda\right|\right)}{\left|\left(\sum_{i=1}^{n} \lambda_{i}^{1}-\alpha_{1}\right) \sum_{i=1}^{n} \lambda_{i}^{2}\right|}\right] \\
& \times\left(\sup _{t \in I}\left|g\left(t, u(t), I^{\beta_{1}} u(t), \ldots, I^{\beta_{n}} u(t)\right)-g\left(t, v(t), I^{\beta_{1}} v(t), \ldots, I^{\beta_{n}} v(t)\right)\right|\right) \\
& =\Omega_{1}\left(\sup _{t \in I}\left|f\left(t, u(t),{ }^{c} D^{\beta_{1}} u(t), \ldots,{ }^{c} D^{\beta_{n}} u(t)\right)-f\left(t, v(t),{ }^{c} D^{\beta_{1}} v(t), \ldots,{ }^{c} D^{\beta_{n}} v(t)\right)\right|\right. \\
& \left.+\sup _{t \in I}\left|g\left(t, u(t), I^{\beta_{1}} u(t), \ldots, I^{\beta_{n}} u(t)\right)-g\left(t, v(t), I^{\beta_{1}} v(t), \ldots, I^{\beta_{n}} v(t)\right)\right|\right) .
\end{aligned}
$$

Let $j \in\{1,2, \ldots, n\}$ be given. Then we have

$$
\begin{aligned}
& \left|{ }^{c} D^{\beta_{j}} T u(t)-{ }^{c} D^{\beta_{j}} T v(t)\right| \\
& =\mid\left\{\frac{1}{\Gamma\left(q-\beta_{j}\right)} \int_{0}^{t}(t-s)^{q-\beta_{j}-1} f\left(s, u(s),{ }^{c} D^{\beta_{1}} u(s), \ldots,{ }^{c} D^{\beta_{n}} u(s)\right) d s\right. \\
& \quad+\frac{\frac{\alpha_{1} t^{1-\beta_{j}}}{\Gamma(q-1)} \int_{0}^{1}(1-s)^{q-2} f\left(s, u(s),{ }^{c} D^{\beta_{1}} u(s), \ldots,{ }^{c} D^{\beta_{n}} u(s)\right) d s}{\Gamma\left(2-\beta_{j}\right)\left(\sum_{i=1}^{n} \frac{a_{i}}{1-\Gamma\left(2-\beta_{i}\right)}-\alpha_{1}\right)\left(\sum_{i=1}^{n}\left(\frac{b_{i}}{\Gamma\left(\beta_{i}+1\right)}\right)\right)}
\end{aligned}
$$




$$
\begin{aligned}
& \left.-\frac{t^{1-\beta_{j}} \sum_{i=1}^{n}\left(\frac{a_{i}}{\Gamma\left(q-\beta_{i}\right)} \int_{0}^{1}(1-s)^{q-\beta_{i}-1} f\left(s, u(s),{ }^{c} D^{\beta_{1}} u(s), \ldots,{ }^{c} D^{\beta_{n}} u(s)\right) d s\right)}{\Gamma\left(2-\beta_{j}\right)\left(\sum_{i=1}^{n} \frac{a_{i}}{1-\Gamma\left(2-\beta_{i}\right)}-\alpha_{1}\right)\left(\sum_{i=1}^{n}\left(\frac{b_{i}}{\Gamma\left(\beta_{i}+1\right)}\right)\right)}\right\} \\
& +\left\{\frac{1}{\Gamma\left(q-\beta_{j}\right)} \int_{0}^{t}(t-s)^{q-\beta_{j}-1} g\left(s, u(s), I^{\beta_{1}} u(s), \ldots, I^{\beta_{n}} u(s)\right) d s\right. \\
& +\frac{\frac{\alpha_{1} t^{1-\beta_{j}}}{\Gamma(q-1)} \int_{0}^{1}(1-s)^{q-2} g\left(s, u(s), I^{\beta_{1}} u(s), \ldots, I^{\beta_{n}} u(s)\right) d s}{\Gamma\left(2-\beta_{j}\right)\left(\sum_{i=1}^{n} \frac{a_{i}}{1-\Gamma\left(2-\beta_{i}\right)}-\alpha_{1}\right)\left(\sum_{i=1}^{n}\left(\frac{b_{i}}{\Gamma\left(\beta_{i}+1\right)}\right)\right)} \\
& \left.-\frac{t^{1-\beta_{j}} \sum_{i=1}^{n}\left(\frac{a_{i}}{\Gamma\left(q-\beta_{i}\right)} \int_{0}^{1}(1-s)^{q-\beta_{i}-1} g\left(s, u(s), I^{\beta_{1}} u(s), \ldots, I^{\beta_{n}} u(s)\right) d s\right)}{\Gamma\left(2-\beta_{j}\right)\left(\sum_{i=1}^{n} \frac{a_{i}}{1-\Gamma\left(2-\beta_{i}\right)}-\alpha_{1}\right)\left(\sum_{i=1}^{n}\left(\frac{b_{i}}{\Gamma\left(\beta_{i}+1\right)}\right)\right)}\right\} \\
& -\left\{\frac{1}{\Gamma\left(q-\beta_{j}\right)} \int_{0}^{t}(t-s)^{q-\beta_{j}-1} f\left(s, v(s),{ }^{c} D^{\beta_{1}} v(s), \ldots,{ }^{c} D^{\beta_{n}} v(s)\right) d s\right. \\
& +\frac{\frac{\alpha_{1} t^{1-\beta_{j}}}{\Gamma(q-1)} \int_{0}^{1}(1-s)^{q-2} f\left(s, v(s),{ }^{c} D^{\beta_{1}} v(s), \ldots,{ }^{c} D^{\beta_{n}} v(s)\right) d s}{\Gamma\left(2-\beta_{j}\right)\left(\sum_{i=1}^{n} \frac{a_{i}}{1-\Gamma\left(2-\beta_{i}\right)}-\alpha_{1}\right)\left(\sum_{i=1}^{n}\left(\frac{b_{i}}{\Gamma\left(\beta_{i}+1\right)}\right)\right)} \\
& \left.-\frac{t^{1-\beta_{j}} \sum_{i=1}^{n}\left(\frac{a_{i}}{\Gamma\left(q-\beta_{i}\right)} \int_{0}^{1}(1-s)^{q-\beta_{i}-1} f\left(s, v(s),{ }^{c} D^{\beta_{1}} v(s), \ldots,{ }^{c} D^{\beta_{n}} v(s)\right) d s\right)}{\Gamma\left(2-\beta_{j}\right)\left(\sum_{i=1}^{n} \frac{a_{i}}{1-\Gamma\left(2-\beta_{i}\right)}-\alpha_{1}\right)\left(\sum_{i=1}^{n}\left(\frac{b_{i}}{\Gamma\left(\beta_{i}+1\right)}\right)\right)}\right\} \\
& -\left\{\frac{1}{\Gamma\left(q-\beta_{j}\right)} \int_{0}^{t}(t-s)^{q-\beta_{j}-1} g\left(s, v(s), I^{\beta_{1}} v(s), \ldots, I^{\beta_{n}} v(s)\right) d s\right. \\
& +\frac{\frac{\alpha_{1} t^{1-\beta_{j}}}{\Gamma(q-1)} \int_{0}^{1}(1-s)^{q-2} g\left(s, v(s), I^{\beta_{1}} v(s), \ldots, I^{\beta_{n}} v(s)\right) d s}{\Gamma\left(2-\beta_{j}\right)\left(\sum_{i=1}^{n} \frac{a_{i}}{1-\Gamma\left(2-\beta_{i}\right)}-\alpha_{1}\right)\left(\sum_{i=1}^{n}\left(\frac{b_{i}}{\Gamma\left(\beta_{i}+1\right)}\right)\right)} \\
& \left.-\frac{t^{1-\beta_{j}} \sum_{i=1}^{n}\left(\frac{a_{i}}{\Gamma\left(q-\beta_{i}\right)} \int_{0}^{1}(1-s)^{q-\beta_{i}-1} g\left(s, v(s), I^{\beta_{1}} v(s), \ldots, I^{\beta_{n}} v(s)\right) d s\right)}{\Gamma\left(2-\beta_{j}\right)\left(\sum_{i=1}^{n} \frac{a_{i}}{1-\Gamma\left(2-\beta_{i}\right)}-\alpha_{1}\right)\left(\sum_{i=1}^{n}\left(\frac{b_{i}}{\Gamma\left(\beta_{i}+1\right)}\right)\right)}\right\} \mid \\
& \leq\left|\frac{1}{\Gamma\left(q-\beta_{j}\right)}\right| \int_{0}^{t}(t-s)^{q-\beta_{j}-1} \mid f\left(s, u(s),{ }^{c} D^{\beta_{1}} u(s), \ldots,{ }^{c} D^{\beta_{n}} u(s)\right) \\
& -f\left(s, v(s),{ }^{c} D^{\beta_{1}} v(s), \ldots,{ }^{c} D^{\beta_{n}} v(s)\right) \mid d s \\
& +\left|\frac{\frac{\alpha_{1} t^{1-\beta_{j}}}{\Gamma(q-1)}}{\Gamma\left(2-\beta_{j}\right)\left(\sum_{i=1}^{n} \frac{a_{i}}{1-\Gamma\left(2-\beta_{i}\right)}-\alpha_{1}\right)\left(\sum_{i=1}^{n}\left(\frac{b_{i}}{\Gamma\left(\beta_{i}+1\right)}\right)\right)}\right| \\
& \times \int_{0}^{1}(1-s)^{q-2} \mid f\left(s, u(s),{ }^{c} D^{\beta_{1}} u(s), \ldots,{ }^{c} D^{\beta_{n}} u(s)\right) \\
& -f\left(s, v(s),{ }^{c} D^{\beta_{1}} v(s), \ldots,{ }^{c} D^{\beta_{n}} v(s)\right) \mid d s \\
& +\left|\frac{1}{\Gamma\left(q-\beta_{j}\right)}\right| \int_{0}^{t}(t-s)^{q-\beta_{j}-1} \mid g\left(s, u(s), I^{\beta_{1}} u(s), \ldots, I^{\beta_{n}} u(s)\right) \\
& -g\left(s, v(s), I^{\beta_{1}} v(s), \ldots, I^{\beta_{n}} v(s)\right) \mid d s \\
& +\left|\frac{\frac{\alpha_{1} t^{1-\beta_{j}}}{\Gamma(q-1)}}{\Gamma\left(2-\beta_{j}\right)\left(\sum_{i=1}^{n} \frac{a_{i}}{1-\Gamma\left(2-\beta_{i}\right)}-\alpha_{1}\right)\left(\sum_{i=1}^{n}\left(\frac{b_{i}}{\Gamma\left(\beta_{i}+1\right)}\right)\right)}\right| \\
& \times \int_{0}^{1}(1-s)^{q-2}\left|g\left(s, u(s), I^{\beta_{1}} u(s), \ldots, I^{\beta_{n}} u(s)\right)-g\left(s, v(s), I^{\beta_{1}} v(s), \ldots, I^{\beta_{n}} v(s)\right)\right| d s
\end{aligned}
$$




$$
\begin{aligned}
\leq & \left(\left|\frac{1}{\Gamma\left(q-\beta_{j}+1\right)}\right|+\left|\frac{\frac{\alpha_{1}}{\Gamma(q)}}{\Gamma\left(2-\beta_{j}\right)\left(\sum_{i=1}^{n} \frac{a_{i}}{1-\Gamma\left(2-\beta_{i}\right)}-\alpha_{1}\right)\left(\sum_{i=1}^{n}\left(\frac{b_{i}}{\Gamma\left(\beta_{i}+1\right)}\right)\right)}\right|\right) \\
& \times\left(\sup _{t \in I}\left|f\left(t, u(t),{ }^{c} D^{\beta_{1}} u(t), \ldots,{ }^{c} D^{\beta_{n}} u(t)\right)-f\left(t, v(t),{ }^{c} D^{\beta_{1}} v(t), \ldots,{ }^{c} D^{\beta_{n}} v(t)\right)\right|\right. \\
& \left.+\sup _{t \in I}\left|g\left(t, u(t), I^{\beta_{1}} u(t), \ldots, I^{\beta_{n}} u(t)\right)-g\left(t, v(t), I^{\beta_{1}} v(t), \ldots, I^{\beta_{n}} v(t)\right)\right|\right) \\
= & \Omega_{2}\left(\sup _{t \in I}\left|f\left(t, u(t),{ }^{c} D^{\beta_{1}} u(t), \ldots,{ }^{c} D^{\beta_{n}} u(t)\right)-f\left(t, v(t),{ }^{c} D^{\beta_{1}} v(t), \ldots,{ }^{c} D^{\beta_{n}} v(t)\right)\right|\right. \\
& \left.+\sup _{t \in I}\left|g\left(t, u(t), I^{\beta_{1}} u(t), \ldots, I^{\beta_{n}} u(t)\right)-g\left(t, v(t), I^{\beta_{1}} v(t), \ldots, I^{\beta_{n}} v(t)\right)\right|\right) .
\end{aligned}
$$

Thus, we get

$$
\begin{aligned}
d(T u, T v) & \\
= & \sup _{t \in I}|T u(t)-T v(t)|+\sup _{t \in I}\left|{ }^{c} D^{\beta_{1}} T u(t)-{ }^{c} D^{\beta_{1}} T v(t)\right|+\cdots \\
& +\sup _{t \in I}\left|{ }^{c} D^{\beta_{n}} T u(t)-{ }^{c} D^{\beta_{n}} T v(t)\right| \\
\leq & \Omega_{1}\left(\sup _{t \in I}\left|f\left(t, u(t),{ }^{c} D^{\beta_{1}} u(t), \ldots,{ }^{c} D^{\beta_{n}} u(t)\right)-f\left(t, v(t),{ }^{c} D^{\beta_{1}} v(t), \ldots,{ }^{c} D^{\beta_{n}} v(t)\right)\right|\right. \\
& \left.+\sup _{t \in I}\left|g\left(t, u(t), I^{\beta_{1}} u(t), \ldots, I^{\beta_{n}} u(t)\right)-g\left(t, v(t), I^{\beta_{1}} v(t), \ldots, I^{\beta_{n}} v(t)\right)\right|\right) \\
& +n \Omega_{2}\left(\sup _{t \in I}\left|f\left(t, u(t),{ }^{c} D^{\beta_{1}} u(t), \ldots,{ }^{c} D^{\beta_{n}} u(t)\right)-f\left(t, v(t),{ }^{c} D^{\beta_{1}} v(t), \ldots,{ }^{c} D^{\beta_{n}} v(t)\right)\right|\right. \\
& \left.+\sup _{t \in I}\left|g\left(t, u(t), I^{\beta_{1}} u(t), \ldots, I^{\beta_{n}} u(t)\right)-g\left(t, v(t), I^{\beta_{1}} v(t), \ldots, I^{\beta_{n}} v(t)\right)\right|\right) \\
= & \left(\Omega_{1}+n \Omega_{2}\right)\left(\sup _{t \in I}\left|f\left(t, u(t),{ }^{c} D^{\beta_{1}} u(t), \ldots,{ }^{c} D^{\beta_{n}} u(t)\right)-f\left(t, v(t),{ }^{c} D^{\beta_{1}} v(t), \ldots,{ }^{c} D^{\beta_{n}} v(t)\right)\right|\right. \\
& \left.+\sup _{t \in I}\left|g\left(t, u(t), I^{\beta_{1}} u(t), \ldots, I^{\beta_{n}} u(t)\right)-g\left(t, v(t), I^{\beta_{1}} v(t), \ldots, I^{\beta_{n}} v(t)\right)\right|\right) \\
\leq & \lambda\left(\sup _{t \in I}|u(t)-v(t)|+\sup _{t \in I}\left|{ }^{c} D^{\beta_{1}} u(t)-{ }^{c} D^{\beta_{1}} v(t)\right|+\cdots+\sup _{t \in I}\left|{ }^{c} D^{\beta_{n}} u(t)-{ }^{c} D^{\beta_{n}} v(t)\right|\right) \\
= & \lambda d(u, v)
\end{aligned}
$$

for all $u, v \in \mathcal{X}$. This implies that $T$ is $\alpha$-contraction. Let $u, v \in \mathcal{X}$ be such that $\alpha(u, v) \geq 1$. Then $\xi\left(u(t),{ }^{c} D^{\beta_{1}} u(t), \ldots,{ }^{c} D^{\beta_{n}} u(t), v(t),{ }^{c} D^{\beta_{1}} v(t), \ldots,{ }^{c} D^{\beta_{n}}\right) \geq 0$ Hence, $\xi\left(T u(t),{ }^{c} D^{\beta_{1}} T u(t)\right.$, $\left.\ldots,{ }^{c} D^{\beta_{n}} T u(t), T v(t),{ }^{c} D^{\beta_{1}} T v(t), \ldots,{ }^{c} D^{\beta_{n}} T v(t)\right) \geq 0$ for all $t \in[0,1]$ and so $\alpha(T u, T v) \geq 1$. It means that $T$ is $\alpha$-admissible. Finally, it is easy to check that $\alpha\left(u_{1}, T u_{1}\right) \geq 1$. Now by using Lemma 1.2, $T$ has approximate fixed point which is an approximate solution for the problem (2.1).

By using Lemma 1.2, one can easily check that the sum-type fractional integrodifferential equation (2.1) has at least one exact solution whenever the functions $f, g$ are continuous.

\section{Numerical method}

In this section, we use the Chebyshev and Legendre polynomials for finding approximate solutions of the problem (2.1). The shifted Chebyshev polynomials be defined 
on $[0,1]$ by $T_{n+1}^{*}(x)=2(2 x-1) T_{n}^{*}(x)-T_{n-1}^{*}(x)$ for all $n \geq 1$, where $T_{1}^{*}(x)=2 x-1$ and $T_{0}^{*}(x)=1$ ([24]). The analytical form of the shifted Chebyshev polynomials $T_{n}^{*}(x)$ is given by $T_{n}^{*}(x)=n \sum_{i=0}^{n}(-1)^{n-i} \frac{2^{2 i}(n+i-1) !}{(2 i) !(n-i) !} x^{i}$ for all $n \geq 1$ ([24]). We have the orthogonality condition $\int_{0}^{1} \frac{T_{n}^{*}(x) T_{m}^{*}(x)}{\sqrt{x-x^{2}}} d x=0$ whenever $m \neq n, \int_{0}^{1} \frac{T_{n}^{*}(x) T_{m}^{*}(x)}{\sqrt{x-x^{2}}} d x=\frac{\pi}{2}$ whenever $m=n \neq 0$ and $\int_{0}^{1} \frac{T_{n}^{*}(x) T_{m}^{*}(x)}{\sqrt{x-x^{2}}} d x=\pi$ whenever $m=n=0$ ([24]). Every function $u \in L^{2}([0,1])$ can be expressed by the shifted Chebyshev polynomials as $u(x)=\sum_{i=0}^{\infty} c_{i} T_{i}^{*}(x)$, where $c_{0}=$ $\frac{1}{\pi} \int_{0}^{1} \frac{u(t) T_{0}^{*}(t)}{\sqrt{t-t^{2}}} d t$ and $c_{i}=\frac{2}{\pi} \int_{0}^{1} \frac{u(t) T_{i}^{*}(t)}{\sqrt{t-t^{2}}} d t$ for all $i \geq 1([22])$. Denote the first $(m+1)$-terms of the shifted Chebyshev polynomials by $u_{m}(x)=\sum_{i=0}^{m} c_{i} T_{i}^{*}(x)$ for all $m \geq 1$ ([22]).

Theorem 3.1 Let $\alpha>0$ be given. Then we have ${ }^{c} D^{\alpha}\left(u_{m}(x)\right)=\sum_{i=\lceil\alpha\rceil}^{m} \sum_{k=\lceil\alpha\rceil}^{i} c_{i} w_{i, k}^{(\alpha)} x^{k-\alpha}$ and $I^{\alpha}\left(u_{m}(x)\right)=\sum_{i=0}^{m} \sum_{k=0}^{i} c_{i} \Theta_{i, k}^{(\alpha)} x^{k+\alpha}$, where $\Theta_{i, k}^{(\alpha)}=(-1)^{i-k} \frac{2^{2 k}(i+k-1) ! \Gamma(k+1)}{(i-k) !(2 k) ! \Gamma(k+1+\alpha)}, \Theta_{0,0}^{(\alpha)}=\frac{1}{\Gamma(\alpha+1)}$ and $w_{i, k}^{(\alpha)}=(-1)^{i-k} \frac{2^{2 k} i(i+k-1) ! \Gamma(k+1)}{(i-k) !(2 k) ! \Gamma(k+1-\alpha)}$.

Proof By using the linear properties of the Caputo fractional derivative, we get

$$
\begin{aligned}
{ }^{c} D^{\alpha}\left(u_{m}(x)\right) & ={ }^{c} D^{\alpha}\left(c_{0} T_{0}^{*}(x)\right)+\sum_{i=1}^{m} c_{i}^{c} D^{\alpha}\left(T_{i}^{*}\right)(x) \\
& ={ }^{c} D^{\alpha}\left(c_{0} T_{0}^{*}(x)\right)+\sum_{i=1}^{m} \sum_{k=0}^{i} c_{i}(-1)^{i-k} \frac{2^{2 k} i(i+k-1) !}{(i-k) !(2 k) !} D^{\alpha}\left(x^{k}\right) .
\end{aligned}
$$

Since ${ }^{c} D^{\alpha}\left(x^{k}\right)=0$ whenever $k=0,1, \ldots,\lceil\alpha\rceil-1$ and ${ }^{c} D^{\alpha}\left(x^{k}\right)=\frac{\Gamma(k+1)}{\Gamma(k+1-\alpha)} x^{k-\alpha}$ whenever $k \geq$ $\lceil\alpha\rceil$, we have

$$
{ }^{c} D^{\alpha}\left(u_{m}(x)\right)=\sum_{i=\lceil\alpha\rceil}^{m} \sum_{k=\lceil\alpha\rceil}^{i} c_{i}(-1)^{i-k} \frac{2^{2 k} i(i+k-1) ! \Gamma(k+1)}{(i-k) !(2 k) ! \Gamma(k+1+\alpha)} x^{k-\alpha}=\sum_{i=\lceil\alpha\rceil}^{m} \sum_{k=\lceil\alpha\rceil}^{i} c_{i} w_{i, k}^{(\alpha)} x^{k-\alpha} .
$$

Also by using the linear properties of the Riemann-Liouville fractional integral, we get

$$
\begin{aligned}
I^{\alpha}\left(u_{m}(x)\right) & =I^{\alpha}\left(c_{0} T_{0}^{*}(x)\right)+\sum_{i=1}^{m} c_{i} I^{\alpha}\left(T_{i}^{*}\right)(x) \\
& =I^{\alpha}\left(c_{0} T_{0}^{*}(x)\right)+\sum_{i=1}^{m} \sum_{k=0}^{i} c_{i}(-1)^{i-k} \frac{2^{2 k} i(i+k-1) !}{(i-k) !(2 k) !} I^{\alpha}\left(x^{k}\right) .
\end{aligned}
$$

Since $I^{\alpha} x^{k}=\frac{\Gamma(k+1)}{\Gamma(k+1+\alpha)} x^{k+\alpha}$, we obtain

$$
\begin{aligned}
I^{\alpha}\left(u_{m}(x)\right) & =\frac{c_{0} x^{k}}{\Gamma(\alpha+1)}+\sum_{i=1}^{m} \sum_{k=0}^{i} c_{i}(-1)^{i-k} \frac{2^{2 k} i(i+k-1) ! \Gamma(k+1)}{(i-k) !(2 k) ! \Gamma(k+1+\alpha)} x^{k+\alpha} \\
& =\sum_{i=0}^{m} \sum_{k=0}^{i} c_{i} \Theta_{i, k}^{(\alpha)} x^{k+\alpha} .
\end{aligned}
$$

This completes the proof.

For solving the problem (2.1) by using the Chebyshev method, we approximate $x(t)$ by

$$
x(t) \cong \sum_{i=0}^{m} c_{i} T_{i}^{*}(t) .
$$


By substituting the estimates (3.1) in (2.1) and applying Theorem 3.1, we obtain

$$
\begin{aligned}
& \sum_{i=\lceil q\rceil}^{m} \sum_{s=\lceil q\rceil}^{i} c_{i} w_{i, s}^{(q)} t^{s-q} \\
& =f\left(t, \sum_{i=0}^{m} c_{i} T_{i}^{*}(t), \sum_{i=\left\lceil\beta_{1}\right\rceil}^{m} \sum_{s=\left\lceil\beta_{1}\right\rceil}^{i} c_{i} w_{i, s}^{\left(\beta_{1}\right)} t^{s-\beta_{1}}, \ldots, \sum_{i=\left\lceil\beta_{n}\right\rceil}^{m} \sum_{s=\left\lceil\beta_{n}\right\rceil}^{i} c_{i} w_{i, s}^{\left(\beta_{n}\right)} t^{s-\beta_{n}}\right) \\
& \quad+g\left(t, \sum_{i=0}^{m} c_{i} T_{i}^{*}(t), \sum_{i=0}^{m} \sum_{s=0}^{i} c_{i} \Theta_{i, s}^{\left(\beta_{1}\right)} t^{s+\beta_{1}}, \sum_{i=0}^{m} \sum_{s=0}^{i} c_{i} \Theta_{i, s}^{\left(\beta_{2}\right)} t^{s+\beta_{2}}, \ldots,\right. \\
& \left.\quad \sum_{i=0}^{m} \sum_{s=0}^{i} c_{i} \Theta_{i, s}^{\left(\beta_{n}\right)} t^{s+\beta_{n}}\right) .
\end{aligned}
$$

In equation (3.2) for $t=x_{p}$ and $p=0, \ldots, m+1-\lceil q\rceil$, we obtain

$$
\begin{aligned}
& \sum_{i=\lceil q\rceil}^{m} \sum_{s=\lceil q\rceil}^{i} c_{i} w_{i, s}^{(q)} x_{p}^{s-q} \\
& =f\left(x_{p}, \sum_{i=0}^{m} c_{i} T_{i}^{*}\left(x_{p}\right), \sum_{i=\left\lceil\beta_{1}\right\rceil} \sum_{s=\left\lceil\beta_{1}\right\rceil}^{i} c_{i} w_{i, s}^{\left(\beta_{1}\right)} x_{p}^{s-\beta_{1}}, \ldots, \sum_{i=\left\lceil\beta_{n}\right\rceil}^{m} \sum_{s=\left\lceil\beta_{n}\right\rceil}^{i} c_{i} w_{i, s}^{\left(\beta_{n}\right)} x_{p}^{s-\beta_{n}}\right) \\
& \quad+g\left(x_{p}, \sum_{i=0}^{m} c_{i} T_{i}^{*}\left(x_{p}\right), \sum_{i=0}^{m} \sum_{s=0}^{i} c_{i} \Theta_{i, s}^{\left(\beta_{1}\right)} x_{p}^{s+\beta_{1}}, \sum_{i=0}^{m} \sum_{s=0}^{i} c_{i} \Theta_{i, s}^{\left(\beta_{2}\right)} x_{p}^{s+\beta_{2}}, \ldots,\right. \\
& \left.\quad \sum_{i=0}^{m} \sum_{s=0}^{i} c_{i} \Theta_{i, s}^{\left(\beta_{n}\right)} x_{p}^{s+\beta_{n}}\right) .
\end{aligned}
$$

For calculating the unknowns $c_{0}, \ldots, c_{m}$, we consider the roots of $T_{m+1-\lceil q\rceil}^{*}(t)$ and use the $\sum_{j=1}^{n}\left(a_{j}^{c} D^{\beta_{j}} x(1)\right)=\alpha_{1} x^{\prime}(1)$ and $\sum_{j=1}^{n}\left(b_{j} I^{\beta_{j}} x(1)\right)=\alpha_{2} x^{\prime}(0)$. Then we get

$$
\sum_{j=1}^{n} a_{j} \sum_{i=\left\lceil\beta_{j}\right\rceil}^{m} \sum_{k=\left\lceil\beta_{j}\right\rceil}^{i} c_{i} w_{i, k}^{\left(\beta_{j}\right)}=\alpha_{1} \sum_{i=1}^{m} \sum_{k=1}^{i} c_{i} w_{i, k}^{(1)}
$$

and

$$
\sum_{j=1}^{n} b_{j} \sum_{i=0}^{m} \sum_{s=0}^{i} c_{i} \Theta_{i, s}^{\left(\beta_{j}\right)}=0 .
$$

Note that equations (3.3) and (3.4) and (3.5) generate $m+1$ nonlinear equations which can be solved by using the Newton iterative method. Thus, we can find the unknowns $c_{0}, \ldots, c_{m}$ and so one can calculate $x(t)$. Similarly, the shifted Legendre polynomials on $[0,1]$ defined by $L_{n+1}^{*}(x)=\frac{(2 n+1)(2 x-1)}{n+1} L_{n}^{*}(x)-\frac{n}{n+1} L_{n-1}^{*}(x)$ for all $n \geq 1$, where $L_{0}^{*}(x)=1$ and $L_{1}^{*}(x)=2 x-1([25])$. In fact, $L_{n}^{*}(x)=\sum_{i=0}^{n}(-1)^{n+i} \frac{(n+i) !}{(n-i) !(i !)^{2}} x^{i}$ for all $n \geq 1, \int_{0}^{1} L_{n}^{*}(x) L_{m}^{*}(x) d x=0$ whenever $m \neq n$ and $\int_{0}^{1} L_{n}^{*}(x) L_{m}^{*}(x) d x=\frac{1}{2 m+1}$ whenever $m=n([25])$. Every function $u \in$ $L^{2}([0,1])$ can be expressed by the shifted Legendre polynomials by $u(x)=\sum_{i=0}^{\infty} c_{i} L_{i}^{*}(x)$, where $c_{i}=(2 i+1) \int_{0}^{1} u(t) L_{i}^{*}(t) d t$ for $i \geq 1([25])$. Denote the first $(m+1)$-terms shifted 
Legendre polynomials by

$$
u_{m}(x)=\sum_{i=0}^{m} c_{i} L_{i}^{*}(x)
$$

By applying a similar proof of Theorem 3.1, one can prove next result.

Theorem 3.2 Let $\alpha>0$ be given. Then we have ${ }^{c} D^{\alpha}\left(u_{m}(x)\right)=\sum_{i=\lceil\alpha\rceil}^{m} \sum_{k=\lceil\alpha\rceil}^{i} c_{i} \mathcal{A}_{i, k}^{(\alpha)} x^{k-\alpha}$ and $I^{\alpha}\left(u_{m}(x)\right)=\sum_{i=0}^{m} \sum_{k=0}^{i} c_{i} \mathcal{B}_{i, k}^{(\alpha)} x^{k+\alpha}, \mathcal{A}_{i, k}^{(\alpha)}=(-1)^{i+k} \frac{(i+k) !}{(i-k) !(k) ! \Gamma(k+1-\alpha)}$ and

$$
\mathcal{B}_{i, k}^{(\alpha)}=(-1)^{i-k} \frac{(i+k) !}{(i-k) !(k) ! \Gamma(k+1+\alpha)} .
$$

Now, we approximate $x(t)$ by

$$
x(t) \cong \sum_{i=0}^{m} d_{i} L_{i}^{*}(t)
$$

By using estimates (3.7) in the problem (2.1) and applying Theorem 3.2, we obtain

$$
\begin{aligned}
\sum_{i=\lceil q\rceil}^{m} & \sum_{s=\lceil q\rceil}^{i} d_{i} \mathcal{A}_{i, s}^{(q)} t^{s-q} \\
= & f\left(t, \sum_{i=0}^{m} d_{i} L_{i}^{*}(t), \sum_{i=\left\lceil\beta_{1}\right\rceil}^{m} \sum_{s=\left\lceil\beta_{1}\right\rceil}^{i} d_{i} \mathcal{A}_{i, s}^{\left(\beta_{1}\right)} t^{s-\beta_{1}}, \ldots, \sum_{i=\left\lceil\beta_{n}\right\rceil}^{m} \sum_{s=\left\lceil\beta_{n}\right\rceil}^{i} d_{i} \mathcal{A}_{i, s}^{\left(\beta_{n}\right)} t^{s-\beta_{n}}\right) \\
& +g\left(t, \sum_{i=0}^{m} d_{i} L_{i}^{*}(t), \sum_{i=0}^{m} \sum_{s=0}^{i} d_{i} \mathcal{B}_{i, s}^{\left(\beta_{1}\right)} t^{s+\beta_{1}}, \sum_{i=0}^{m} \sum_{s=0}^{i} d_{i} \mathcal{B}_{i, s}^{\left(\beta_{2}\right)} t^{s+\beta_{2}}, \ldots\right. \\
& \left.\sum_{i=0}^{m} \sum_{s=0}^{i} d_{i} \mathcal{B}_{i, s}^{\left(\beta_{n}\right)} t^{s+\beta_{n}}\right) .
\end{aligned}
$$

Now, we collocate (3.8) at $m+1-\lceil q\rceil$ points $x_{p}(p=0, \ldots, m+1-\lceil q\rceil)$ as

$$
\begin{aligned}
\sum_{i=\lceil q\rceil}^{m} & \sum_{s=\lceil q\rceil}^{i} d_{i} \mathcal{A}_{i, s}^{(q)} x_{p}^{s-q} \\
= & f\left(t, \sum_{i=0}^{m} d_{i} L_{i}^{*}\left(x_{p}\right), \sum_{i=\left\lceil\beta_{1}\right\rceil}^{m} \sum_{s=\left\lceil\beta_{1}\right\rceil}^{i} d_{i} \mathcal{A}_{i, s}^{\left(\beta_{1}\right)} x_{p}^{s-\beta_{1}}, \ldots, \sum_{i=\left\lceil\beta_{n}\right\rceil}^{m} \sum_{s=\left\lceil\beta_{n}\right\rceil}^{i} d_{i} \mathcal{A}_{i, s}^{\left(\beta_{n}\right)} x_{p}^{s-\beta_{n}}\right) \\
& +g\left(x_{p}, \sum_{i=0}^{m} d_{i} L_{i}^{*}\left(x_{p}\right), \sum_{i=0}^{m} \sum_{s=0}^{i} d_{i} \mathcal{B}_{i, s}^{\left(\beta_{1}\right)} x_{p}^{s+\beta_{1}}, \sum_{i=0}^{m} \sum_{s=0}^{i} d_{i} \mathcal{B}_{i, s}^{\left(\beta_{2}\right)} x_{p}^{s+\beta_{2}}, \ldots,\right. \\
& \left.\sum_{i=0}^{m} \sum_{s=0}^{i} d_{i} \mathcal{B}_{i, s}^{\left(\beta_{n}\right)} x_{p}^{s+\beta_{n}}\right),
\end{aligned}
$$

where $x_{p}(p=0, \ldots, m+1-\lceil q\rceil)$ are roots of the polynomial $P_{m+1-\lceil q\rceil}^{*}(t)$. Also by substituting equation (3.7), Theorem 3.2 and the conditions $\sum_{j=1}^{n}\left(a_{j}^{c} D^{\beta_{j}} x(1)\right)=\alpha_{1} x^{\prime}(1)$ and 


$$
\begin{aligned}
& \sum_{j=1}^{n}\left(b_{j} I^{\beta_{j}} x(1)\right)=\alpha_{2} x^{\prime}(0) \text {, we get } \\
& \qquad \sum_{j=1}^{n} a_{j} \sum_{i=\left\lceil\beta_{j}\right\rceil}^{m} \sum_{k=\left\lceil\beta_{j}\right\rceil}^{i} d_{i} \mathcal{A}_{i, k}^{\left(\beta_{j}\right)}=\alpha_{1} \sum_{i=1}^{m} \sum_{k=1}^{i} d_{i} \mathcal{A}_{i, k}^{(1)}
\end{aligned}
$$

and

$$
\sum_{j=1}^{n} b_{j} \sum_{i=0}^{m} \sum_{s=0}^{i} d_{i} \mathcal{B}_{i, s}^{\left(\beta_{j}\right)}=0
$$

Note that equations (3.9) and (3.10) and (3.11) generate $m+1$ nonlinear equations which can be solved by using the Newton iterative method to obtain the unknown $d_{0}, \ldots, d_{m}$. Thus, one can calculate the solution $x(t)$ of the problem. Here, we provide two examples to illustrate our numerical methods. There is much work which provides some methods for numerical solutions of some types fractional differential equations (see [11, 14] and [18]). Our aim is not to introduce a method that can be answered with greater accuracy and speed. The following examples illustrate our main results and we show that numerical approximations could be exact sometimes.

Example 1 Consider the fractional differential equation

$$
\begin{aligned}
{ }^{c} D^{\frac{3}{2}} x(t)= & {[10 t+\sin (t)]+\ln (|\sinh (t)|+1)+\frac{1}{20}\left(x(t)+{ }^{c} D^{\frac{1}{3}} x(t)\right) } \\
& +\left[{ }^{c} D^{\frac{1}{2}} x(t)+0.5\right]
\end{aligned}
$$

with the boundary conditions ${ }^{c} D^{\frac{1}{2}} x(1)+{ }^{c} D^{\frac{1}{3}} x(1)=x^{\prime}(1)$ and $I^{\frac{1}{2}} x(1)+I^{\frac{1}{3}} x(1)=x^{\prime}(0)$. Consider the function $f\left(t, x_{1}, x_{2}, x_{3}\right)=[10 t+\sin (t)]+\ln (|\sinh (t)|+1)+\frac{x_{1}}{20}+\left[x_{2}+0.5\right]+\frac{x_{3}}{20}$, $g\left(t, x_{1}, x_{2}, x_{3}\right)=0$ and $\xi\left(\left(x_{1}, x_{2}, x_{3}\right),\left(y_{1}, y_{2}, y_{3}\right)\right)=1$ whenever $x_{2}=0$ and $y_{2}=0$ almost everywhere and $\xi\left(\left(x_{1}, x_{2}, x_{3}\right),\left(y_{1}, y_{2}, y_{3}\right)\right)=-1$ otherwise. Put $n=2, \lambda=0.9, \alpha_{1}=\alpha_{2}=a_{1}=$ $a_{2}=b_{1}=b_{2}=1, \beta_{1}=\frac{1}{2}, \beta_{2}=\frac{1}{3}$ and $q=\frac{3}{2}$. One can check that the problem (3.12) satisfy the conditions of Theorem (2.2), where, thus, the problem (3.12) has an approximate solution. Check Tables 1 and 2 and Figure 1.

One can find the coefficients $c_{i}$ and $d_{i}$ by using the explained Chebyshev and Legendre methods as in Table 1. Also, one can find difference of the numerical approximate solutions in Figure 1 and Table 2. Here, we denote the numerical solutions of the Chebyshev and Legendre methods by $\tilde{x}$ and $\hat{x}$, respectively.

Table 1 Coefficients

\begin{tabular}{lcc}
\hline $\boldsymbol{i}$ & Coefficient value of Chebyshev method $\boldsymbol{c}_{\boldsymbol{i}}$ & Coefficient value of Legendre method $\boldsymbol{d}_{\boldsymbol{i}}$ \\
\hline 1 & -13.64912481 & -13.89585847 \\
2 & 32.68664752 & 32.61068585 \\
3 & 0.739732907 & 0.983914814 \\
4 & 0.123903993 & 0.188170898 \\
5 & 0.004270165 & 0.012299691 \\
6 & 0.011334926 & 0.02302969 \\
7 & -0.004503085 & -0.009980864 \\
\hline
\end{tabular}


Table 2 Differences

\begin{tabular}{ll}
\hline $\boldsymbol{t}$ & $|\tilde{\boldsymbol{x}}(\boldsymbol{t})-\hat{\boldsymbol{x}}(\boldsymbol{t})|$ \\
\hline 0.0 & $4.76063632959267 \mathrm{e}-13$ \\
0.1 & $3.90798504668055 \mathrm{e}-13$ \\
0.2 & $3.26849658449646 \mathrm{e}-13$ \\
0.3 & $2.62900812231237 \mathrm{e}-13$ \\
0.4 & $1.98951966012828 \mathrm{e}-13$ \\
0.5 & $1.36779476633819 \mathrm{e}-13$ \\
0.6 & $7.19424519957101 \mathrm{e}-14$ \\
0.7 & $8.88178419700125 \mathrm{e}-15$ \\
0.8 & $5.59552404411079 \mathrm{e}-14$ \\
0.9 & $1.26121335597418 \mathrm{e}-13$ \\
1 & $1.84741111297626 \mathrm{e}-13$ \\
\hline
\end{tabular}

Figure 1 Chebyshev and Legendre method.

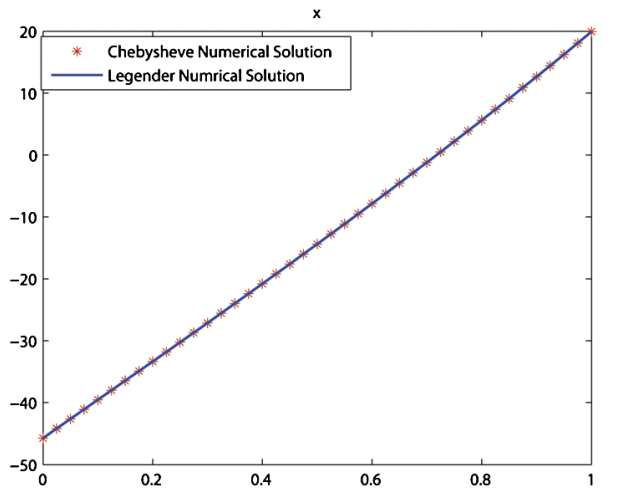

Example 2 Consider the fractional integro-differential equation

$$
\begin{aligned}
{ }^{c} D^{\frac{\sqrt{6}}{2}} x(t)= & e^{\cos (t)}+\ln (t+1)+\ln \left(t^{2}+1\right)+\frac{1}{30}\left(\left(t+t^{5}\right) x(t)+t^{2 c} D^{\frac{1}{2}} x(t)+\frac{\sqrt{t}}{t+1} D^{\frac{1}{3}} x(t)\right. \\
& +\left(\frac{\sin x(t)}{\sin ^{2} x(t)+1}\right){ }^{c} D^{\frac{\sqrt{3}}{2}} x(t)-\frac{2 I^{\frac{\sqrt{3}}{2}} x(t)}{2\left|I^{\frac{\sqrt{3}}{2}} x(t)\right|+3} I^{\frac{1}{2}} x(t)+\frac{\sqrt[5]{t}}{9} I^{\frac{1}{3}} x(t) \\
& \left.-\frac{1}{4} I^{\frac{\sqrt{3}}{2}} x(t)\right)
\end{aligned}
$$

with boundary conditions $-{ }^{c} D^{\frac{1}{2}} x(1)+{ }^{c} D^{\frac{1}{3}}=2 x^{\prime}(1)$ and $2 I^{\frac{1}{2}} x(1)-3 I^{\frac{\sqrt{3}}{2}} x(1)=x^{\prime}(0)$. Consider the continuous functions

$$
f\left(t, x_{1}, x_{2}, x_{3}, x_{4}\right)=e^{\cos (t)}+\ln (t+1)+\frac{1}{30}\left(t x_{1}+t^{2} x_{2}+\frac{\sqrt{t}}{t+1} x_{3}+\frac{\sin x(t)}{\sin ^{2} x(t)+1} x_{4}\right)
$$

and $g\left(t, x_{1}, x_{3}, x_{3}, x_{4}\right)=\ln \left(t^{2}+1\right)+\frac{1}{30}\left(t^{5} x_{1}-\frac{2 x_{3} x_{2}}{2\left|x_{3}\right|+3}+\frac{\sqrt[5]{t} x_{3}}{9}-\frac{x_{4}}{2}\right)$. Define the map $\xi\left(\left(x_{1}, x_{2}\right.\right.$, $\left.\left.x_{3}, x_{4}\right),\left(y_{1}, y_{2}, y_{3}, y_{4}\right)\right)=1$ for all $x_{1}, \ldots, x_{4}, y_{1}, \ldots, y_{4} \in \mathbb{R}$. Put $n=3, \lambda=0.9, a_{1}=-1, a_{2}=1$, $a_{3}=0, b_{1}=2, b_{2}=0, b_{3}=-3, \alpha_{1}=2, \alpha_{2}=1, \beta_{1}=\frac{1}{2}, \beta_{2}=\frac{1}{3}, \beta_{3}=\frac{\sqrt{3}}{2}$ and $q=\frac{\sqrt{6}}{2}$. Thus by using Theorem 2.2, the problem (3.13) has an exact solution. Check Tables 3 and 4 and Figure 2. We present the coefficients $c_{0}, c_{1}, \ldots, c_{6}$ and $d_{0}, d_{1}, \ldots, d_{6}$ (for $m=6$ ) by using the Chebyshev and Legendre methods in Table 3. As one easily sees, the difference of the numerical approximate solutions by the Chebyshev and Legendre methods (which 
Table 3 Coefficients

\begin{tabular}{lcc}
\hline $\boldsymbol{i}$ & Coefficient value of Chebyshev method $\boldsymbol{c}_{\boldsymbol{i}}$ & Coefficient value of Legendre method $\boldsymbol{d}_{\boldsymbol{i}}$ \\
\hline 0 & 25.0832696245301 & 25.0923764343546 \\
1 & -100.27385020664 & -100.245026343328 \\
2 & -0.023785620114085 & -0.0164038330046405 \\
3 & -0.045007244673989 & -0.0606901595074366 \\
4 & -0.0234838615675559 & -0.0564629360395388 \\
5 & -0.0127366109683591 & -0.0258775587930064 \\
6 & 0.0135562287330535 & 0.030046706107713 \\
\hline
\end{tabular}

Table 4 Differences

\begin{tabular}{ll}
\hline $\boldsymbol{t}$ & $|\tilde{\boldsymbol{x}}(\boldsymbol{t})-\hat{\boldsymbol{x}}(\boldsymbol{t})|$ \\
\hline 0 & $8.17294676380698 \mathrm{E}-10$ \\
0.1 & $6.89240664542012 \mathrm{E}-10$ \\
0.2 & $5.59495560992218 \mathrm{E}-10$ \\
0.3 & $4.26595647695649 \mathrm{E}-10$ \\
0.4 & $2.9262992029544 \mathrm{E}-10$ \\
0.5 & $1.60159885354005 \mathrm{E}-10$ \\
0.6 & $3.02238234439756 \mathrm{E}-11$ \\
0.7 & $9.81437153768638 \mathrm{E}-11$ \\
0.8 & $2.27224461468722 \mathrm{E}-10$ \\
0.9 & $3.58717500148487 \mathrm{E}-10$ \\
1 & $4.90501861349912 \mathrm{E}-10$ \\
\hline
\end{tabular}

Figure 2 Chebyshev and Legendre method.

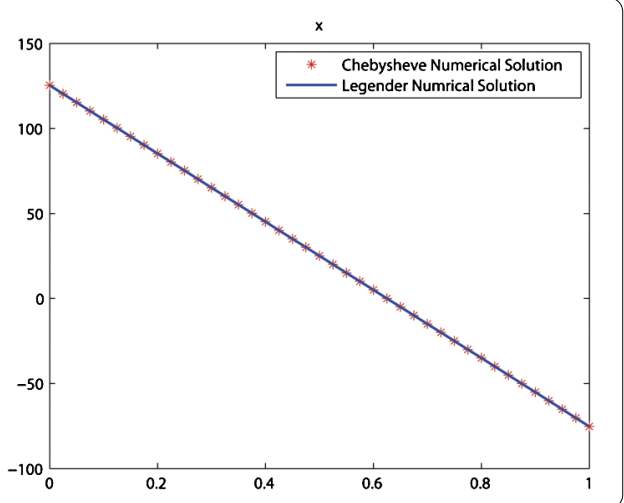

has been provided in Figure 2) is inconsiderable. Denote the numerical solutions of the Chebyshev and Legendre methods by $\tilde{x}$ and $\hat{x}$, respectively. In Table 4 , we show that the difference of the approximate solutions obtained by Chebyshev and Legendre methods is negligible.

\section{Conclusions}

We first prove the existence of approximate solutions for a sum-type fractional integrodifferential problem via Caputo differentiation. By using the shifted Legendre and Chebyshev polynomials, we provide a numerical method for finding solutions for the problem. Also, we give two examples to illustrate our results from a numerical point of view. Our aim is not to introduce a method that can be answered with greater accuracy and speed. 


\section{Acknowledgements}

The authors would like to thank the esteemed referees for their important comments, which improved the final version of this manuscript. The research of the authors was supported by Azarbaijan Shahid Madani University.

\section{Competing interests}

The authors declare that they have no competing interests.

\section{Authors' contributions}

All authors have made equal contributions. The whole work was carried out, read and approved by the authors.

\section{Publisher's Note}

Springer Nature remains neutral with regard to jurisdictional claims in published maps and institutional affiliations.

\section{Received: 19 June 2017 Accepted: 17 October 2017 Published online: 02 November 2017}

\section{References}

1. Reinermann, J: Uber Toeplitzsche Iterationsverfahren und einige ihrer Anwendungen in der konstruktiven Fixpunkttheorie. Stud. Math. 32, 209-227 (1969) (in German)

2. Yamamoto, Y, Ohtsubo, H: Subspace iteration accelerated by using Chebyshev polynomials for eigenvalue problems with symmetric matrices. Int. J. Numer. Methods Eng. 10(4), 935-944 (1976)

3. Khader, MM: Introducing an efficient modification of the variational iteration method by using Chebyshev polynomials. Appl. Appl. Math. 7(1), 283-299 (2012)

4. Khader, MM: Introducing an efficient modification of the homotopy perturbation method by using Chebyshev polynomials. Arab J. Math. Sci. 18(1), 61-71 (2012)

5. Maleknejad, K, Sohrabi, S, Rostami, Y: Numerical solution of nonlinear Volterra integral equations of the second kind by using Chebyshev polynomials. Appl. Math. Comput. 188, 123-128 (2007)

6. Chen, YM, Liu, LL, Sun, L, Sun, XH: Numerical solution of a nonlinear fractional integro-differential equation by using Legendre wavelets. J. Hefei Univ. Technol. Nat. Sci. 36(8), 1019-1024 (2013) (in Chinese)

7. Mirzaee, F, Fathi, S: Numerical solution of some class of integro-differential equations by using Legendre-Bernstein basis. J. Hyperstruct. 3(2), 139-154 (2014)

8. Yousefi, SA: Numerical solution of Abel's integral equation by using Legendre wavelets. Appl. Math. Comput. 175, 574-580 (2006)

9. Zhou, FY, Xu, XY: Numerical approximation of definite integrals by using Legendre wavelets and operator matrices. Acta Math. Appl. Sin. 38(5), 862-873 (2015)

10. Aydogan, SM, Baleanu, D, Mousalou, A, Rezapour, Sh: On approximate solutions for two higher-order Caputo-Fabrizio fractional integro-differential equations. Adv. Differ. Equ. 2017, 221 (2017)

11. Bhrawy, AH, Zaky, MA: Shifted fractional-order Jacobi orthogonal functions: application to a system of fractional differential equations. Appl. Math. Model. 40(2), 832-845 (2016)

12. Jafarian, A, Rostami, F, Golmankhaneh, AK, Baleanu, D: Using ANNs approach for solving fractional order Volterra integro-differential equations. Int. J. Comput. Intell. Syst. 10(1), 470-480 (2017)

13. Kumar, D, Singh, J, Baleanu, D: Modified Kawahara equation within a fractional derivative with non-singular kernel. Therm. Sci. (2017). doi:10.2298/TSCI160826008K

14. Mashayekhi, S, Razzaghi, M: Numerical solution of distributed order fractional differential equations by hybrid functions. J. Comput. Phys. 315, 169-181 (2016)

15. Singh, J, Kumar, D, Nieto, JJ: A reliable algorithm for a local fractional Tricomi equation arising in fractal transonic flow. Entropy 2016, 206 (2016)

16. Singh, J, Kumar, D, Al Qurashi, M, Baleanu, D: A new fractional model for giving up smoking dynamics. Adv. Differ. Equ. 2017, 88 (2017)

17. Singh, J, Kumar, D, Al Qurashi, M, Baleanu, D: A novel numerical approach for a nonlinear fractional dynamical model of interpersonal and romantic relationships. Entropy 2017, 375 (2017)

18. Singh, H, Srivastava, HM, Kumar, D: A reliable numerical algorithm for the fractional vibration equation. Chaos Solitons Fractals 103, 131-138 (2017)

19. Suganya, S, Baleanu, D, Kalamani, P, Arjunan, MM: A note on non-instantaneous impulsive fractional neutral integro-differential systems with state-dependent delay in Banach spaces. J. Comput. Anal. Appl. 20, 1302-1317 (2016)

20. Kilbas, AA, Srivastava, HM, Trujillo, JJ: Theory and Applications of Fractional Differential Equations, vol. 204. Elsevier, Amsterdam (2006)

21. Podlubny, I: Fractional Differential Equations. Academic Press, San Diego (1999)

22. Miandaragh, MA, Postolache, M, Rezapour, Sh: Some approximate fixed point results for generalized $\alpha$-contractive mappings. Sci. Bull. "Politeh." Univ. Buchar., Ser. A, Appl. Math. Phys. 75(2), 3-10 (2013)

23. Su, X: Boundary value problem for a coupled system of nonlinear fractional differential equations. Appl. Math. Lett. 22, 64-69 (2009)

24. Snyder, MA: Chebyshev Methods in Numerical Approximation. Prentice Hall, Englewood Cliffs (1966)

25. Khader, MM: Numerical solution of nonlinear multi-order fractional differential equations by implementation of the operational matrix of fractional derivative. Stud. Nonlinear Sci. 2(1), 5-12 (2011) 